\title{
Helminth-induced arginase-1 exacerbates lung inflammation and disease severity in tuberculosis
}

\author{
Leticia Monin, ${ }^{1,2}$ Kristin L. Griffiths, ${ }^{2}$ Wing Y. Lam, ${ }^{3}$ Radha Gopal, ${ }^{1}$ Dongwan D. Kang,,${ }^{4,5}$ Mushtaq Ahmed, ${ }^{2}$ \\ Anuradha Rajamanickam, ${ }^{6}$ Alfredo Cruz-Lagunas, ${ }^{7}$ Joaquín Zúñiga, ${ }^{7}$ Subash Babu, ${ }^{6}$ Jay K. Kolls, ${ }^{8}$ \\ Makedonka Mitreva, ${ }^{9,10,11}$ Bruce A. Rosa, ${ }^{9}$ Rosalio Ramos-Payan, ${ }^{12,13}$ Thomas E. Morrison, ${ }^{14}$ \\ Peter J. Murray, ${ }^{15}$ Javier Rangel-Moreno, ${ }^{12}$ Edward J. Pearce, ${ }^{3}$ and Shabaana A. Khader ${ }^{1,2}$
}

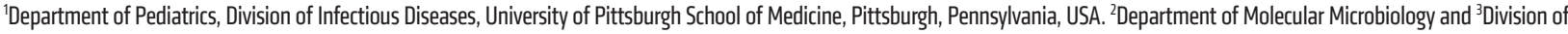
Immunobiology, Department of Pathology and Immunology, Washington University School of Medicine, St. Louis, Missouri, USA. ${ }^{4}$ Department of Energy, Joint Cenome Institute, Walnut Creek, California, USA. ${ }^{5}$ Cenomics Division, Lawrence Berkeley National Laboratory, Berkeley, California, USA. ${ }^{6}$ National Institutes of Health - National Institute for Research in TuberculosisInternational Center for Excellence (NIH-NIRT-ICER), Chennai, India. 'Instituto Nacional de Enfermedades Respiratorias "Ismael Cosio Villegas", Mexico City, Mexico. ${ }^{8}$ Richard King Mellon Foundation Institute for Pediatric Research, Children's Hospital of Pittsburgh of UPMC, Pittsburgh, Pennsylvania, USA. ${ }^{9}$ The Genome Institute, ${ }^{10}$ Division of Infectious Diseases, Department of Internal Medicine, and "Department of Cenetics, Washington University School of Medicine, St. Louis, Missouri, USA. '2Department of Medicine, Division of Allergy, Immunology and Rheumatology, University of Rochester Medical Center, Rochester, New York, USA. ${ }^{3}$ Faculty of Biological and Chemical Sciences, Autonomous University of Sinaloa, Culiacan, Sinaloa, Mexico. ${ }^{14}$ Department of Immunology and Microbiology, University of Colorado School of Medicine, Aurora, Colorado, USA. ${ }^{15}$ Departments of Infectious Diseases and Immunology, St. Jude Children's Research Hospital, Memphis, Tennessee, USA.

Parasitic helminth worms, such as Schistosoma mansoni, are endemic in regions with a high prevalence of tuberculosis (TB) among the population. Human studies suggest that helminth coinfections contribute to increased TB susceptibility and increased rates of TB reactivation. Prevailing models suggest that T helper type 2 (Th2) responses induced by helminth infection impair Th1 immune responses and thereby limit Mycobacterium tuberculosis (Mtb) control. Using a pulmonary mouse model of Mtb infection, we demonstrated that S. mansoni coinfection or immunization with S. mansoni egg antigens can reversibly impair $M t b$-specific T cell responses without affecting macrophage-mediated $M t b$ control. Instead, S. mansoni infection resulted in accumulation of high arginase-1-expressing macrophages in the lung, which formed type 2 granulomas and exacerbated inflammation in Mtb-infected mice. Treatment of coinfected animals with an antihelminthic improved Mtb-specific Th1 responses and reduced disease severity. In a genetically diverse mouse population infected with Mtb, enhanced arginase-1 activity was associated with increased lung inflammation. Moreover, in patients with pulmonary TB, lung damage correlated with increased serum activity of arginase-1, which was elevated in TB patients coinfected with helminths. Together, our data indicate that helminth coinfection induces arginase-1-expressing type 2 granulomas, thereby increasing inflammation and TB disease severity. These results also provide insight into the mechanisms by which helminth coinfections drive increased susceptibility, disease progression, and severity in TB.

\section{Introduction}

About one-third of the world's population is infected with $\mathrm{Myco}$ bacterium tuberculosis ( $M t b)$, leading to 1.4 million deaths and 8.8 million new reported cases of pulmonary tuberculosis (TB) every year (1). Although most $M t b$-infected people have latent TB (LTB) and remain asymptomatic, they have a $10 \%$ lifetime risk of developing active TB (ATB). Mtb infection induces a potent $\mathrm{T}$ helper type 1 (Th1) immune response in the host, which contributes to protective immunity (2). Interestingly, helminth parasites, which characteristically elicit Th2 responses $(3,4)$, are endemic in the populations where TB prevalence is highest (5). Approximately 1 billion people are infected with one or more helminths, of which schistosomiasis, caused by trematodes of the genus Schistosoma mansoni (S. mansoni), affects about 207 million individuals (6). Human studies on coinfected individuals suggest that infection

Conflict of interest: The authors have declared that no conflict of interest exists. Submitted: January 30, 2015; Accepted: October 8, 2015.

Reference information: J Clin Invest. 2015;125(12):4699-4713. doi:10.1172/JCI77378. with helminths compromise protection conferred by $M$. bovis Bacillus Calmette-Guerin (BCG) vaccination (7-9) and may be associated with increased TB reactivation rates in HIV-infected patients (10). In experimental models, mice chronically infected with $S$. mansoni have increased susceptibility to M. bovis BCG systemic infection, accompanied by decreased M. bovis BCG-specific Th1 responses (11). Additionally, $S$. mansoni-infected mice vaccinated with $M$. bovis BCG demonstrate reduced protection upon subsequent $M t b$ systemic challenge (12). Thus, the prevailing models suggest that Th2 responses induced due to helminth coinfections impair Th1 immune responses and $M t b$ control.

Using a mouse model of $M t b$ infection, we show that coinfection with a helminth, such as $S$. mansoni, or immunization with $S$. mansoni-derived egg antigens can reversibly impair $M t b$ specific Th1 cell responses without severely affecting the ability of the macrophages to limit $M t b$ control. Importantly, S. mansoni infection results in accumulation of arginase-1-expressing macrophages in the lung, forming type 2 granulomas and resulting in increased lung fibrosis and mucous plug formation, and exac- 
erbating disease severity and inflammation in $M t b$-coinfected mice. These inflammatory effects are mediated by macrophages expressing arginase- 1 and can be reversed by treatment with antihelminthics. Our recent work has put forth the new paradigm that protective outcomes in TB are associated with formation of lymphoid follicles within the tubercle granuloma (13), while increased disease severity is associated with accumulation of neutrophils and exacerbated inflammation (14). We show that an outcome of $S$. mansoni infection and the induction of type 2 granulomas within the $M t b$-infected lung is the reduced formation of lymphoid follicle-containing granulomas in helminth/Mtb-coinfected hosts and increased accumulation of inflammatory granulocytes. In addition, using $M t b$-infected genetically diverse mice, which exhibit heterogeneity in inflammation and disease severity (14), we demonstrate that exacerbated lung inflammation - even in the absence of helminth coinfection - is associated with increased arginase- 1 activity. Importantly, increased arginase- 1 activity in the serum of patients with ATB is associated with exacerbated lung inflammatory damage and disease severity. Increased arginase- 1 activity detected in the serum from helminth-coinfected ATB patients when compared with ATB patients without helminth coinfections further substantiates our findings. Thus, our data together put forth the hypothesis that helminth coinfection or exposure to helminth antigens increases TB susceptibility and disease severity by induction of arginase-1-expressing macrophages and formation of inflammatory granulomas, rather than just impairing Th1 responses and $M t b$ control. These data provide insights into the mechanisms by which helminth coinfections increase susceptibility, accelerate disease progression, and facilitate reactivation of $\mathrm{TB}$.

\section{Results}

S. mansoni coinfection induces lung fibrosis and exacerbates inflammation during TB. Although helminth coinfections are thought to increase susceptibility to $M t b$ infection, the mechanisms underlying this association remain poorly understood. To experimentally model helminth and $M t b$ coinfection, we infected C57BL/6 arginase-1 yellow fluorescent protein (YFP) reporter mice (Yarg mice) with $S$. mansoni and allowed helminth infection to progress for 6 weeks, a period associated with induction of potent Th2 responses (15). Following this, mice were infected with low doses of aerosolized $M t b$, and TB disease progression was monitored. Mice coinfected with S. mansoni and $M t b$ had larger inflammatory granulomas in the lungs (Figure 1A and Supplemental Figure 1A, upper panel; supplemental material available online with this article; doi:10.1172/JCI77378DS1), which contained both eosinophils and neutrophils (Supplemental Figure 1A, lower panel). In contrast, smaller and more lymphocytic granulomas were detected in the lungs of $M t b$-infected mice (Figure $1 \mathrm{~A}$ and Supplemental Figure 1A). The increased inflammatory response coincided with a significant increase in the number of arginase-1-expressing macrophages in $S$. mansoni/Mtb-coinfected mice, when compared with $M t b$-infected mice (Figure 1B). In addition, increased pulmonary mRNA expression for Arg1, and increased arginase-1 activity in the serum of $S$. mansoni/Mtb-coinfected mice was detected, when compared with $M t b$-infected mice (Figure 1B). Interestingly, in coinfected lungs, we found that type 2 granulomas containing high arginase-1-expressing macrophages were distinct (55\% of granulomas) and in close proximity to type 1 granulomas $(45 \%$ of granulomas) typically containing macrophages expressing high inducible nitric oxide synthase (iNOS) (Figure 1, C and D), while no type 2 granulomas were observed in $M t b$-infected lungs. In addition, arginase-1-expressing macrophages were also localized within the lung interstitium of $S$. mansoni/Mtb-coinfected mice but not in the lungs of $M t b$-infected mice (Figure 1, C and D). Interestingly, mice infected with $S$. mansoni alone also exhibited increased inflammation (Supplemental Figure 1B), when compared with either uninfected lungs or $M t b$-infected lungs and exhibited increased accumulation of arginase-1-expressing macrophages (Figure 1C, Supplemental Figure 1C). F4/80-expressing macrophages (Figure 1C) - and not T cells, B cells, or eosinophils - were the predominant expressors of arginase- 1 within the tubercle granulomas in the lungs of coinfected mice (Supplemental Figure 1D). Furthermore, using Yarg-coinfected mice, we found that lung macrophages - but not neutrophils, eosinophils, or lymphocytes - were the major producers of arginase- 1 in coinfected mice (Supplemental Figure 1E). Purified lung macrophages also expressed higher arginase-1 mRNA levels when compared with neutrophils isolated from coinfected mice (Supplemental Figure $1 F)$. Together, these data demonstrate that $S$. mansoni infection results in accumulation of arginase-1-expressing macrophages in the lung and, upon infection with $M t b$, promotes exacerbated inflammation and increased TB disease severity.

Coincident with increased accumulation of arginase-1expressing macrophages, we also found increased expression of Th2 cytokines such as Il4 and Il13 mRNA in the lungs of S. mansoni/Mtb-coinfected mice (Figure 2A). This also coincided with increased expression of mRNA for matrix metalloproteases, such as $M m p 13$, and neutrophil-derived products, such as myeloperoxidases (Mpo), in S. mansoni/Mtb-coinfected lungs (Figure 2A). Furthermore, lungs from S. mansoni/Mtb-coinfected mice showed increased numbers of Muc5 $\mathrm{AC}^{+}$goblet cells, increased staining for mucins and glycogen in Periodic acid-Schiff-stained (PAS-stained) lung sections, and increased collagen deposition within type 2 granulomas (Figure 2B). These data together suggest that helminth infection drives accumulation of high arginase-1expressing macrophages inside type 2 granulomas and interstitial areas, induces mucus production and local fibrosis, and exacerbates pulmonary inflammation in $M t b$-coinfected mice.

Our recent studies have demonstrated that, in $\mathrm{TB}$, protective granulomas contain distinct B cell lymphoid follicles harboring $\mathrm{T}$ cells and macrophages, while inflammatory granulomas lack well-formed B cell-containing lymphoid follicles and instead contain neutrophils that mediate inflammation $(13,14)$. Thus, we next measured the area occupied by lymphoid B cell follicles and found that $S$. mansoni coinfection in $M t b$-infected mice resulted in decreased and poorly formed B cell follicles (Figure 2C). Consistent with reduced $\mathrm{B}$ cell follicle formation, we also found that $\mathrm{T}$ cell accumulation was decreased within the lymphoid follicles, and $\mathrm{T}$ cells instead formed perivascular cuffs (Figure 2D). These data suggest that the majority of granulomas formed in $S$. mansoni/Mtb-coinfected lungs are preferentially composed of granulocytes and loosely organized B cell lymphoid follicles, a feature associated with nonprotective inflammatory granulomas (13). 
A

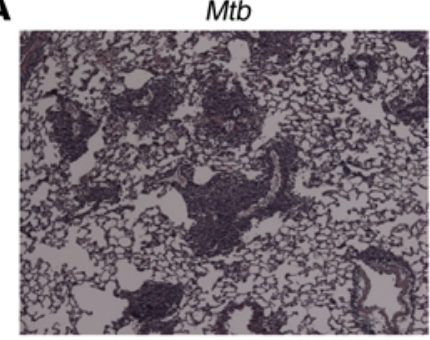

B

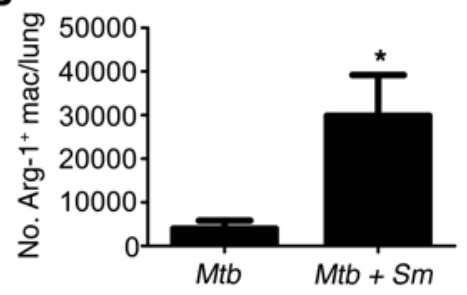

C

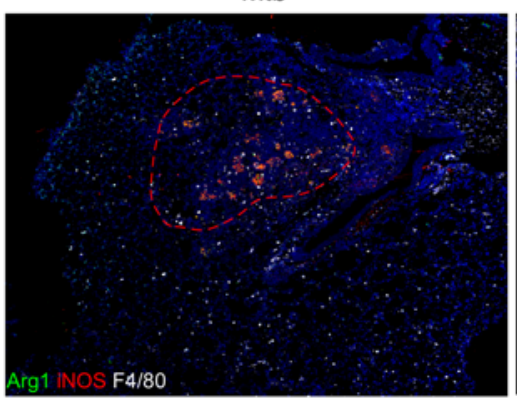

$\mathrm{Mtb}+\mathrm{Sm}$
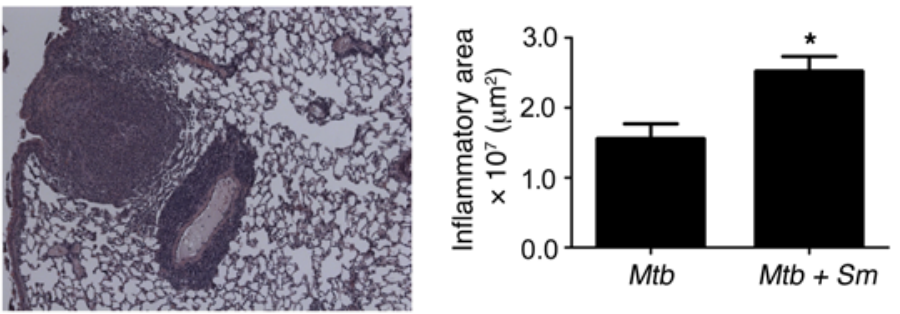

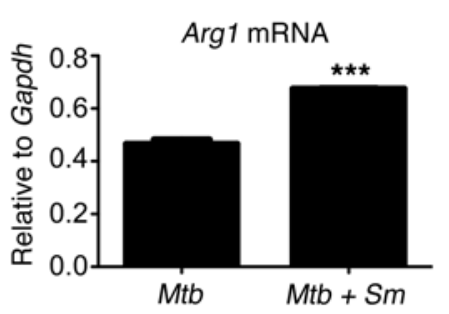

$M t b+S m$
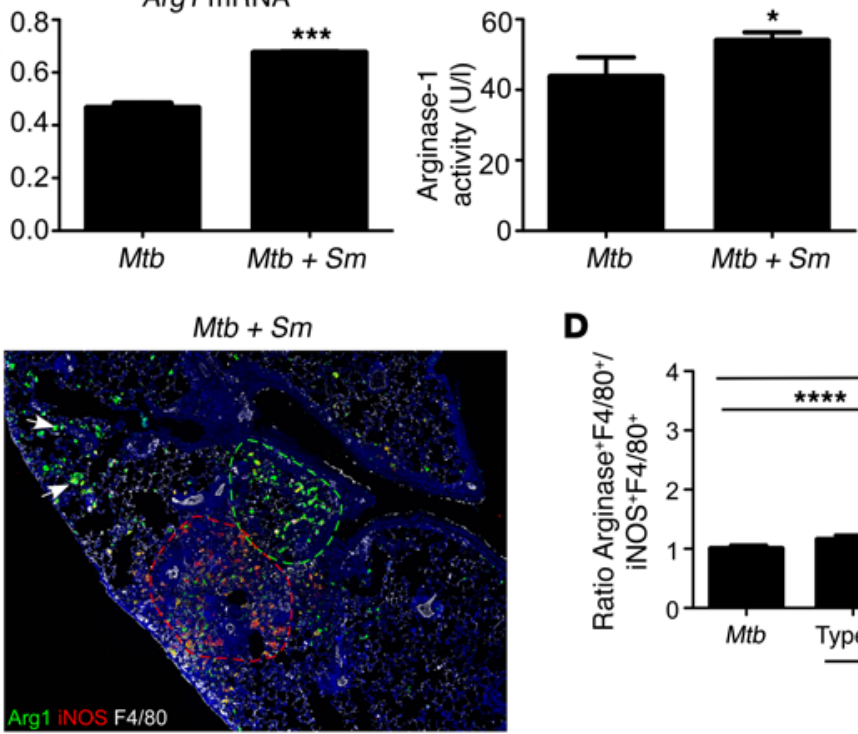

D

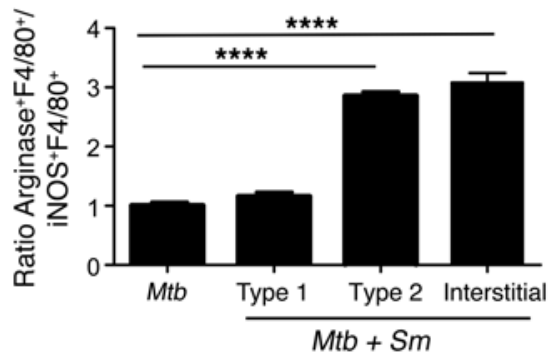

Figure 1. S. mansoni induces arginase-1-expressing type $\mathbf{2}$ granulomas and exacerbates inflammation during coinfection with Mtb. S. mansoniinfected $(M t b+S m)$ or control Yarg mice $(M t b)$ were aerosol infected with $M t b$. (A) On d30 after infection, pulmonary inflammation was assessed on formalin-fixed, paraffin embedded (FFPE) lung sections stained with H\&E. Magnification: $\times 100$. Total area occupied by inflammatory lesions per lobe was quantified. (B) Arginase-1 reporter expression in lung macrophages was detected by flow cytometry (left panel). Arg1 mRNA expression in total lung was assessed by RT-PCR and expression relative to Gapdh expression shown (middle panel). Arginase-1 activity was quantitated in the serum of infected mice (right panel). (C) FFPE sections from infected mice underwent immunofluorescence staining using antibodies specific for arginase-1, iNOS, and F4/80. Mosaics (4×4) of $\times 200$ magnification fields are shown. Type 1 granulomas (red selection), type 2 granulomas (green selection), and interstitial macrophages (arrows) are shown. (D) The number of high iNOS+ and high arginase-1-expressing F4/80+ cells per $\times 200$ field were counted, and ratio of high arginase-1-expressing cells to high iNOS-expressing cells per field is shown. $n=4-5$ mice per group. Lungs of all mice were included in the analysis; one representative image per group is shown. ${ }^{*} P \leq 0.05,{ }^{* *} P \leq 0.001,{ }^{* * *} P \leq 0.0001$ unpaired; 2 -tailed Student's $t$ test (A and $\left.\mathbf{B}\right)$ or 1 -way ANOVA with posthoc Tukey (D) were used. Results are representative of 3 independent experiments.

S. mansoni coinfection reversibly impairs $T h 1$ responses and limits Mtb bacterial control. Accumulation of IFN- $\gamma$-expressing Th1 cells is critical for protective immunity against $M t b$ infection (16). We found that accumulation of IFN- $\gamma$-producing activated CD $4^{+}$ T cells was impaired in $S$. mansoni/Mtb-coinfected lungs (Figure $2 \mathrm{E}$ ), and this coincided with a trend in the increase in IL-4-producing $\mathrm{CD}^{+} \mathrm{T}$ cells (Figure $2 \mathrm{~F}$ ) and no differences in accumulation of Foxp3-expressing Tregs (data not shown). In addition, the impaired Th1 response was associated with decreased activation of macrophages, as measured by MHC Class II (MHCII) expression (Figure 2G). Interestingly, the small but significant impairment in production of IFN- $\gamma$ by lung $\mathrm{CD}^{+} \mathrm{T}$ cells in $S$. mansoni/Mtb-coinfected mice (Figure $2 \mathrm{E}$ ) was reversible, as the number of $M t b$-specific IFN- $\gamma$-producing $C D 4^{+} \mathrm{T}$ cells measured by in vitro overnight antigen-driven ELISpot assay was comparable between $S$. mansoni/Mtb-coinfected mice and $M t b$-infected mice (Figure $2 \mathrm{H}$ ). Furthermore, we found that the impairment in Th1 responses ex vivo coincided with a significant but slight increase in lung $M t b$ burden in S. mansoni/Mtb-coinfected mice when compared with $M t b$-infected mice (Figure 2I). Unexpectedly, our data thus suggest that the Th2 responses induced by helminth infection have reversible effects on Th1 immunity and minimal effect on mycobacterial control, but they more prominently affect lung inflammation.

Treatment of S. mansoni/Mtb-coinfected mice with an antihelminthic can reverse TB disease severity. Praziquantel is an antihelminthic used for treatment of human schistosomiasis (17). Thus, we next addressed if treatment with an antihelminthic such as praziquantel can be used to limit disease severity in $S$. mansoni/Mtb-coinfected mice. Mice were infected with $S$. mansoni and coinfected with $M t b$, following which one group was treated with praziquantel while the control coinfected group was treated with PBS. Treatment with praziquantel resulted in significantly decreased S. mansoni egg burden in the liver (Supplemental Figure 

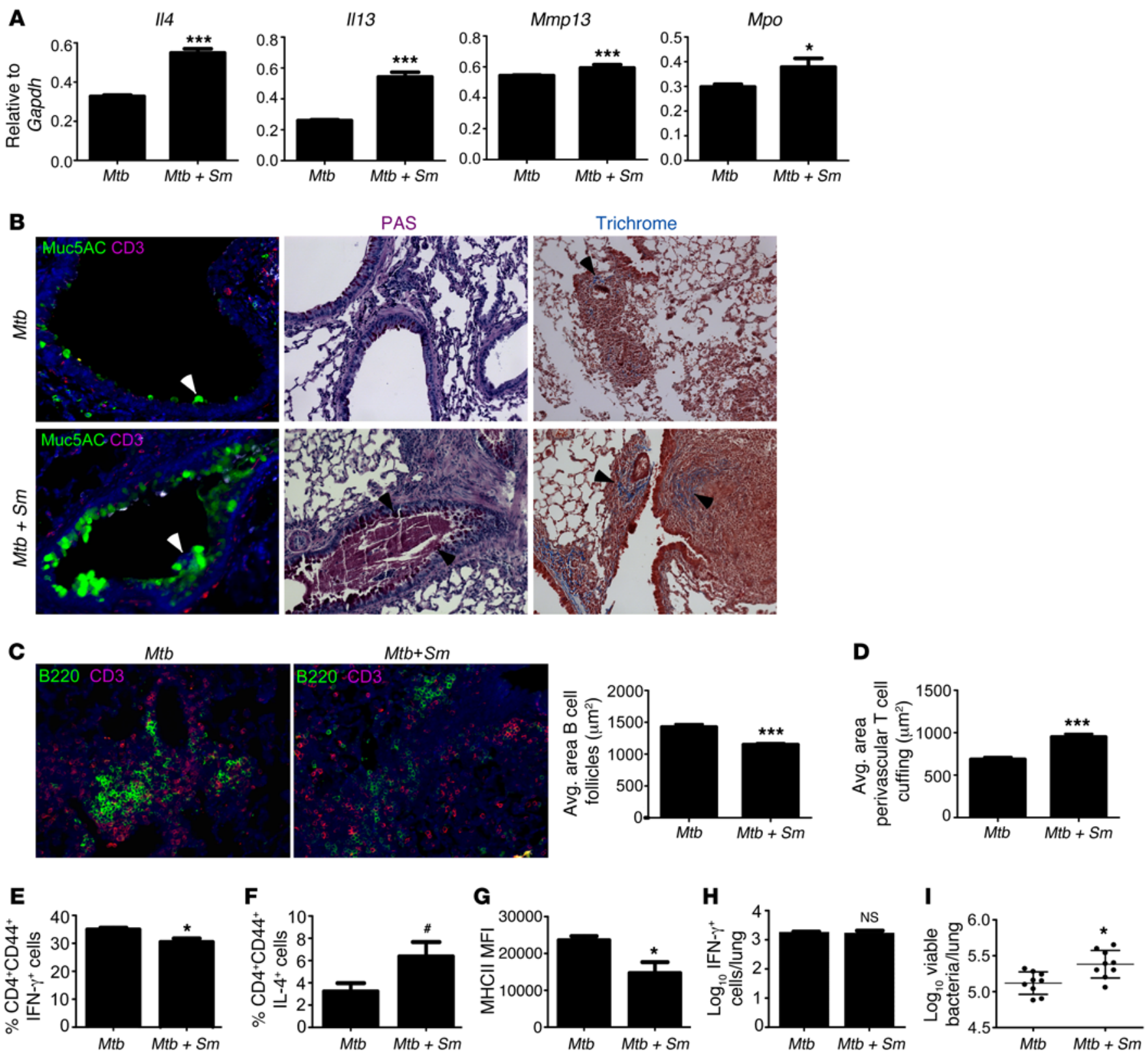

Figure 2. S. mansoni coinfection leads to increased lung pathology, impaired Th1 responses, and increased susceptibility to Mtb infection. S. mansoniinfected $(M t b+S m)$ or control Yarg mice $(M t b)$ were infected with $M t b$. (A) On d30 after infection, lung expression of 114 , I113, Mmp13, and Mpo mRNA relative to Gapdh was determined by RT-PCR. (B) Formalin-fixed, paraffin embedded (FFPE) sections were processed for immunofluorescence using antibodies for Muc5AC and CD3; mucus and glycogen accumulation by the PAS stain and collagen deposition by Gomori's Trichrome stain are shown. Magnification: $\times 200$. Arrows indicate areas of positive staining. (C-D) Immunofluorescence staining for B220 and CD3 was also performed. Magnification: $\times 200$. The average size of $B$ cell follicles within granulomas $(\mathbf{C})$ and the average area of perivascular $T$ cell cuffing $(\mathbf{D})$ were calculated. (E-F) The percentage of lung IFN- $\gamma-(E)$ and IL-4-producing activated T cells (CD4+CD44+IFN- $\gamma^{+}$and CD4+CD44+IL-4+, respectively) (F) were determined by flow cytometry. (C) MHCIl expression on lung macrophages was determined by flow cytometry. (H) The percentage of ESAT- $6_{1-20}-$ specific, IFN- $\gamma$-producing cells in the lungs was determined by antigen-driven ELISpot. (I) Lung bacterial burden was determined by plating. $n=4-9$ mice per group. Lungs of all mice were included in the analysis; one representative image per group is shown. ${ }^{\#} P=0.0616,{ }^{*} P \leq 0.05,{ }^{* *} P \leq 0.001$; unpaired, 2 -tailed Student's $t$ test (A and C-I). Results are representative of 2 independent experiments.

2A). As before, S. mansoni/Mtb-coinfected control mice exhibited increased disease severity (Figure 3, A and B) and increased collagen deposition (Supplemental Figure 2B), when compared with $M t b$-infected mice. Importantly, coinfected mice treated with praziquantel significantly reversed exacerbated inflammation (Figure 3, A and B), decreased collagen deposition within pulmo- nary granulomas (Supplemental Figure 2B), decreased staining for Muc5 $\mathrm{AC}^{+}$goblet cells, and decreased accumulation of mucins and glycogen (Supplemental Figure 2, C and D). The decreased inflammation in praziquantel-treated coinfected mice coincided with a reduction in serum arginase- 1 activity (Figure 3C) and decreased accumulation of high arginase-1-expressing macrophages within 


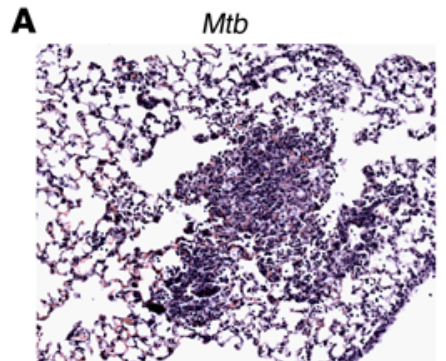

B

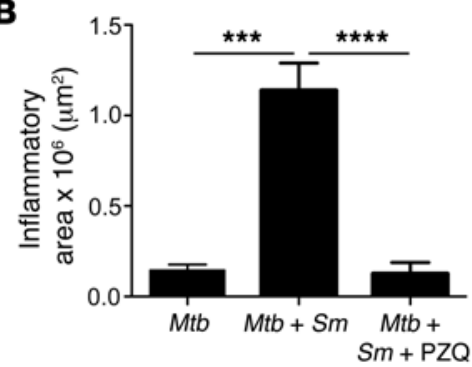

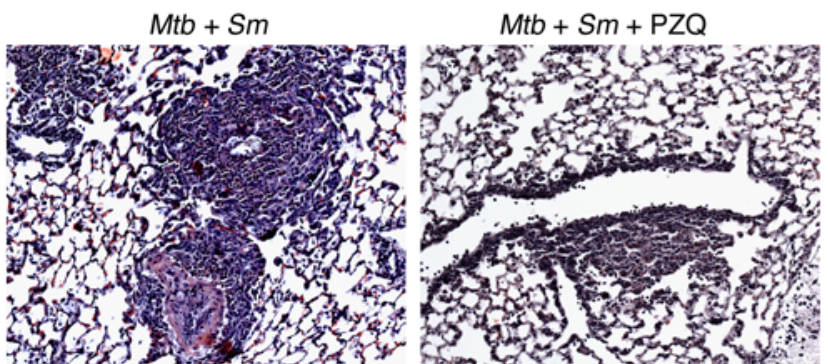

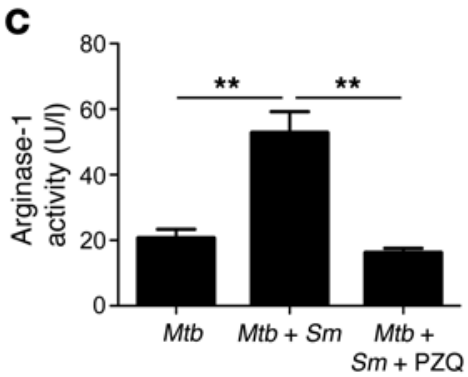

D
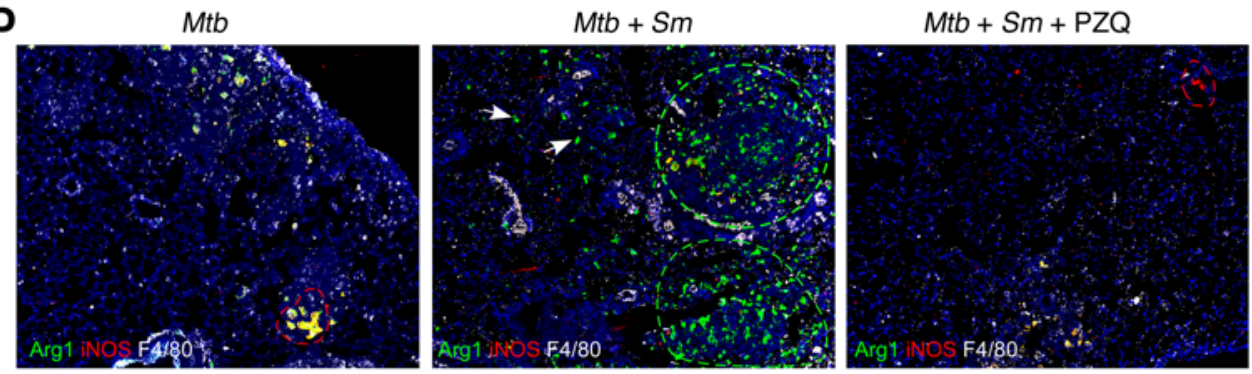

E
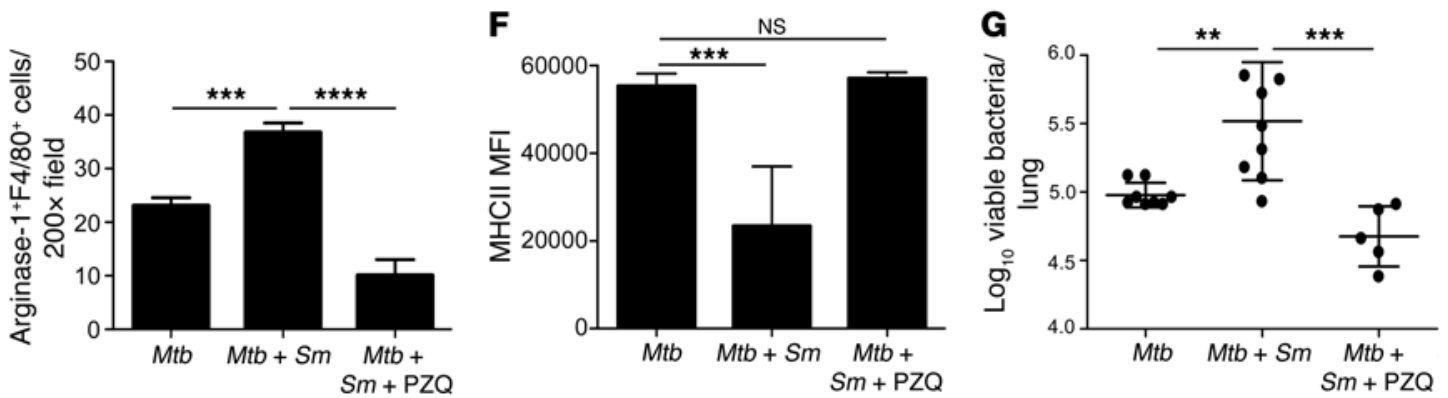

Figure 3. Praziquantel treatment of S. mansoni infection reverses the increased inflammation seen in Mtb/S. mansoni-coinfected mice. S. mansoniinfected $(M t b+S m)$ or control C57BL/6 mice $(M t b)$ were aerosol infected with Mtb and treated with praziquantel (Mtb + Sm + PZQ). (A) On d30 after infection, pulmonary inflammation was assessed on formalin-fixed, paraffin embedded (FFPE) lung sections stained with H\&E. Magnification: $\times 200$. (B) Total area occupied by inflammatory lesions per lobe was quantified. (C) Arginase-1 activity was quantitated in the serum of infected mice. (D) FFPE sections from infected mice underwent immunofluorescence staining using antibodies specific for arginase-1, iNOS, and F4/80. Mosaics (4×4) of $\times 200$ magnification fields are shown. Type 1 granulomas (red selection), type 2 granulomas (green selection), and interstitial macrophages (arrows) are shown. (E) The number of high arginase-1-expressing $\mathbf{F} 4 / 80^{+}$cells per $\times 200$ field was determined. (F) MHCIl expression on lung macrophages was determined by flow cytometry. (C) Lung bacterial burden was determined by plating. $n=4-5$ mice per group. Lungs of all mice were included in the analysis; one representative image per group is shown. ${ }^{* *} P \leq 0.01,{ }^{* *} P \leq 0.001,{ }^{* * *} P \leq 0.0001$; one-way ANOVA with post-hoc Tukey (B, C, and E-G).

the type 2 granulomas in the lungs (Figure 3, D and E). This also coincided with increased activation of lung macrophages (Figure $3 F$ ) and improved $M t b$ control (Figure 3G) in praziquantel-treated coinfected mice when compared with control coinfected mice. Together, these data provide formal evidence that use of antihelminthics can reverse the accumulation of arginase-1-expressing macrophages in the lung to prevent exacerbated inflammation and decrease disease severity in S. mansoni/Mtb-coinfected hosts.
Arginase-1 expression drives exacerbated inflammation in helminth/Mtb-coinfected hosts. Our data thus far suggest that $S$. mansoni infection induces the accumulation of arginase-1-expressing macrophages within the lung, which mediate exacerbated inflammation and increased TB disease upon $M t b$ infection. To mechanistically address if arginase- 1 expression in macrophages mediates the exacerbated disease seen in helminth/Mtb-coinfected mice, we then generated BM chimeric mice (BMC mice). 
A

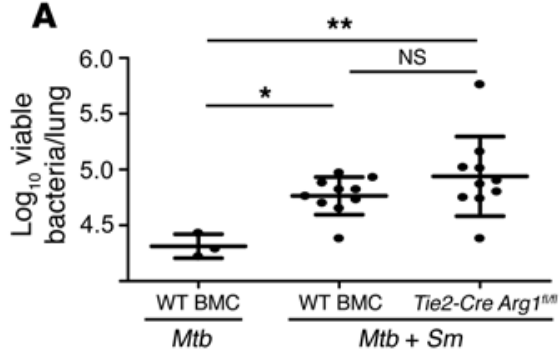

B

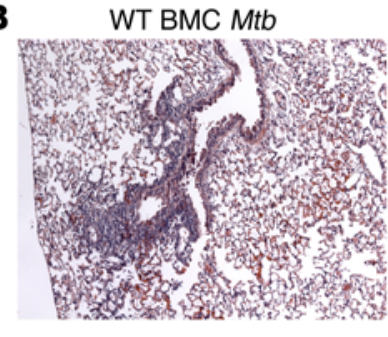

WT BMC Mtb + Sm

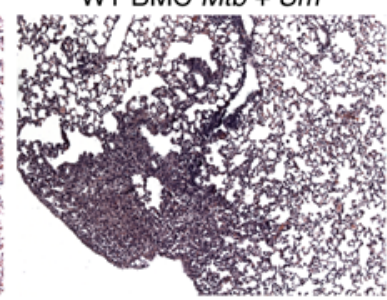

Tie2-Cre Arg $1^{\text {fll }} \mathrm{Mtb}+\mathrm{Sm}$

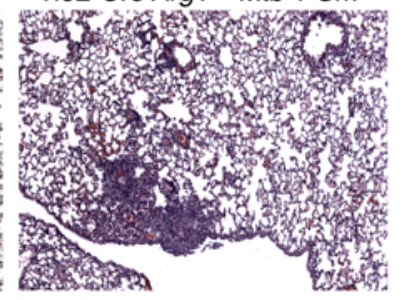

C

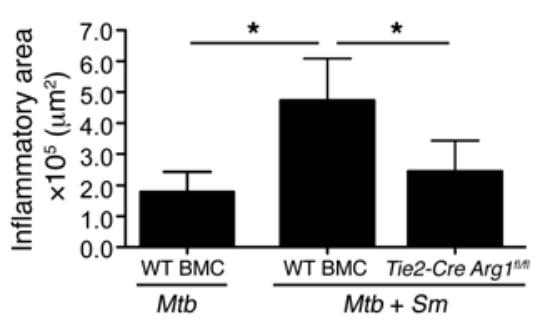

D $\quad \mathrm{WT} B M C M t b+S m$

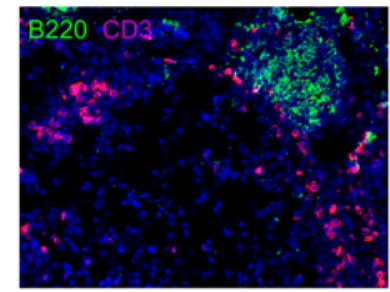

Tie2-Cre Arg ${ }^{\text {thit }} \mathrm{Mtb}+\mathrm{Sm}$

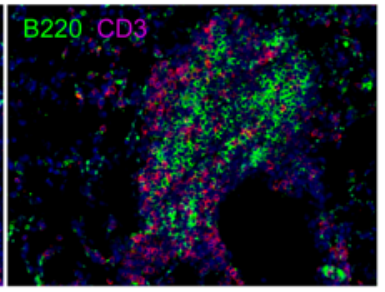

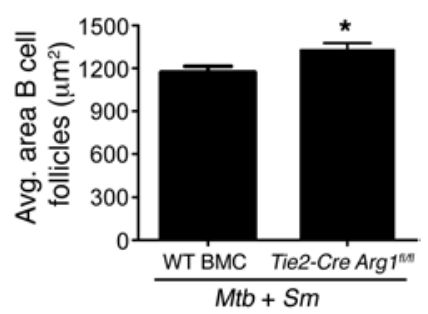

\section{E}

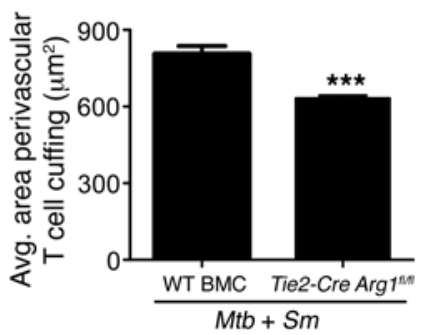

F

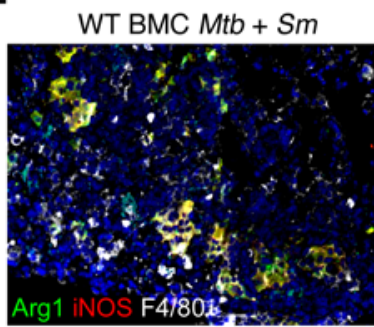

Tie2-Cre Arg $1^{\text {thII }} \mathrm{Mtb}+\mathrm{Sm}$
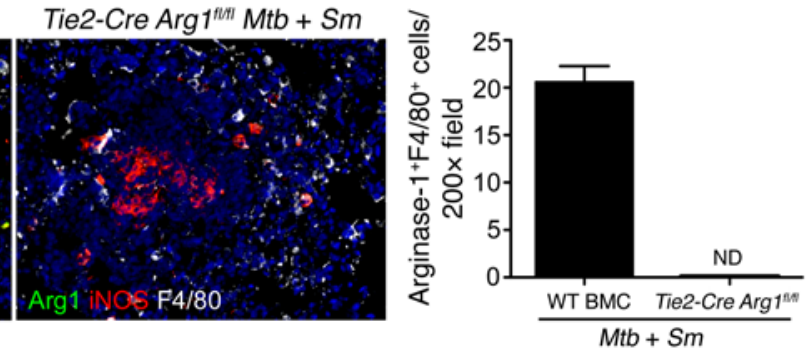

Figure 4. Absence of arginase-1 in lung macrophages reverses lung pathology upon coinfection. WT BMC or Tie2-Cre Arg $7^{f / f f}$ BMC mice were S. mansoni/ $M t b$ coinfected $(M t b+S m)$ or infected with $M t b$ alone $(M t b)$. (A) On d25 after infection, lung bacterial burden was determined by plating. (B) Pulmonary inflammation was assessed on formalin-fixed, paraffin embedded (FFPE) lung sections stained with H\&E. Magnification: $\times 100$. (C) Total area occupied by inflammatory lesions per lobe was quantified. (D) FFPE sections were processed for immunofluorescence using antibodies for B220 and CD3. Magnification: $\times 200$. The average size of B cell follicles within granulomas was determined. (E) The average area of perivascular T cell cuffing in B220- and CD3-stained sections was calculated. (F) FFPE sections were stained with antibodies specific for arginase-1, iNOS, and F4/80. Magnification: $\times 200$. The number of high arginase-1-expressing $\mathrm{F} 4 / 80^{+}$cells per $\times 200$ field was determined. $n=3-10$ mice per group. Lungs of all mice were included in the analysis; one representative image per group is shown. ${ }^{*} P \leq 0.05$, ${ }^{* *} P \leq 0.01$, ${ }^{* * *} P \leq 0.001$. ND, not detectable. One-way ANOVA with post-hoc Tukey (A and $\mathbf{C}$ ) and unpaired, 2-tailed Student's $t$ tests were used (D-F).

The Tie2-Cre deleter is active in all hematopoietic cells, but as arginase- 1 is predominantly expressed in macrophages in TB and schistosomiasis $(18,19)$, use of Tie2-Cre Arg $1^{f l / l} \mathrm{BM}$ cells for reconstitution of BMC will delete arginase- 1 in macrophages (18-22). In addition, as only BM myeloid cells lacking arginase-1 were transferred, any effects of host-derived endothelial arginase-1 are avoided. Thus, C57BL/6.SJL host mice received either WT BM (WT BMC-Arg $1^{f / f l}$ ) or BM from arginase-1-deficient mice where myeloid cells, including macrophages, are devoid of arginase (Tie2-Cre Arg1 fl/fl BMC) (18). Upon confirmation of complete reconstitution, BMC mice were infected with $S$. mansoni, followed by coinfection with $M t b$ as before. Under these conditions, both WT BMC and Tie2-Cre Arg1 fl/fl BMC-coinfected mice exhibited a slight but significant increase in bacterial burden when compared with $M t b$-infected WT BMC mice (Figure $4 \mathrm{~A}$ ). In contrast, Tie2-Cre Arg1 $f^{l / f l} \mathrm{BMC}$-coinfected mice exhibited decreased lung inflammation (Figure 4, B and C), increased formation of protective B cell follicles within TB granulomas (Figure 4D), and decreased $\mathrm{T}$ cell perivascular cuffing when compared with WT BMC-coinfected mice (Figure 4E). This coincided with the complete absence of arginase-1-expressing macrophages within the lungs of Tie2-Cre Arg $1^{f / f l}$ BMC-coinfected mice, when compared with accumulation of high arginase-1-expressing macrophages in lungs of WT BMC-coinfected mice (Figure 4F). To provide additional confirmation that macrophages are the primary producers of arginase- 1 in coinfected mice, we also generated BMC mice where C57BL/6.SJL host mice received either WT BMC-Arg $1^{f / f l}$ or BM from Lysm-Cre Arg1 $1^{f l f l}$ mice (deletes in monocytes, macrophages, and neutrophils). As before (18), while macrophages generated from BM cells from Tie2-Cre Arg1 $1^{f / f l}$ mice expressed no detectable arginase-1 protein, macrophages generated from BM cells from Lysm-Cre Arg $1^{f / f l}$ mice expressed substantially reduced levels of arginase-1 expression (Supplemental Figure 3, A and B). However, similar to the Tie2-Cre Arg $1^{f / f l} \mathrm{BMC}$-coinfected mice, the Lysm-Cre Arg ${ }^{f l / f l} \mathrm{BMC}-$ coinfected mice also demonstrated higher bacterial burden (Supplemental Figure 4A). In addition, also sim- 
A

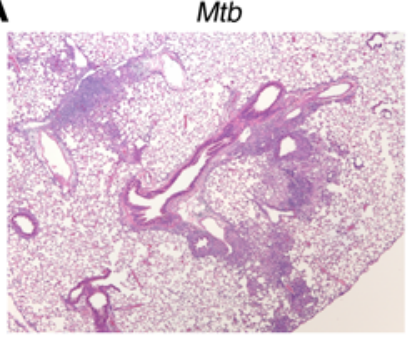

B

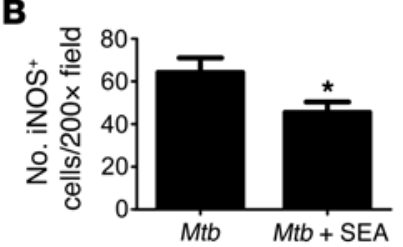

D

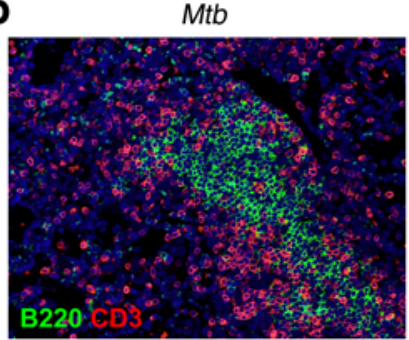

$M t b+$ SEA

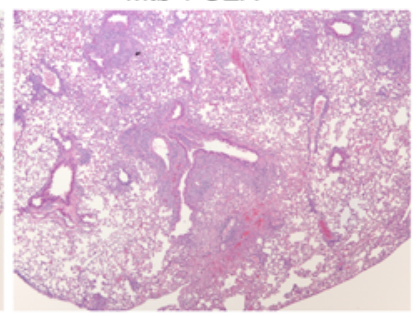

C
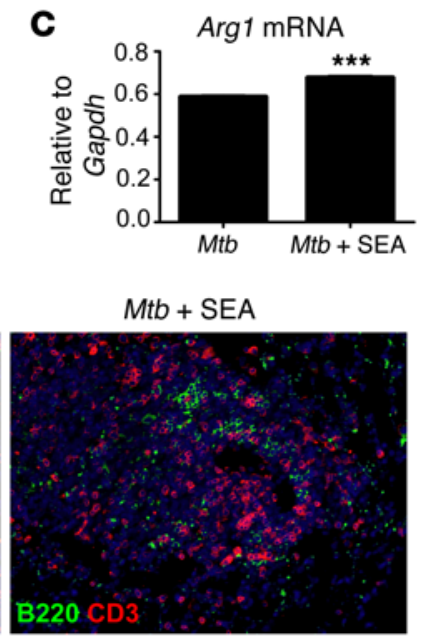
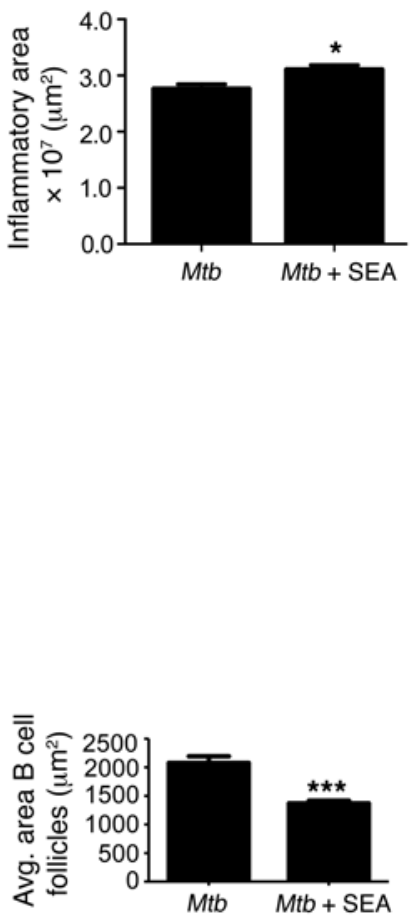

E

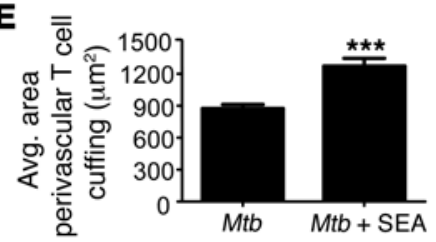

Figure 5. SEA immunization increases susceptibility to Mtb infection. C57BL/6 mice were aerosol infected with $M t b$ and immunized with SEA (Mtb + SEA) or saline (Mtb) on d15 after infection. (A) Pulmonary inflammation was assessed on d30 after infection on $\mathrm{H} \& \mathrm{E}$-stained formalin-fixed, paraffin embedded (FFPE) lung sections. Magnification: $\times 40$. Total area occupied by inflammatory lesions per lobe was quantified.

(B) FFPE sections from infected lungs were processed for immunofluorescence using antibodies specific for iNOS and $\mathrm{F} 4 / 80$, and the number of iNOSexpressing $\mathrm{F} 4 / 80^{+}$cells per $200 \times$ field was counted. (C) CD11C cells were sorted from the lungs of SEA-treated Mtbinfected mice and Mtb-infected mice, and level of Arg1 mRNA relative to Gapdh was determined by RT-PCR. (D-E) FFPE lung sections were also stained using antibodies specific for B220 and CD3. Magnification: $\times 200$. The average size of B cell follicles within granulomas (D) and the average area occupied by perivascular T cell cuffing (E) were calculated. $n=4-8$ mice per group. Lungs of all mice were included in the analysis; one representative image per group is shown. ${ }^{*} P \leq 0.05,{ }^{* *} P \leq 0.001$; unpaired, 2-tailed Student's $t$ test. Results are representative of 2 independent experiments. ilar to the Tie2-Cre Arg1 $1^{f / f l} \mathrm{BMC}$-coinfected mice, Lysm-Cre Arg1 $1^{f / f l}$ coinfected BMC exhibited decreased inflammation (Supplemental Figure 4, B and C), and this coincided with improved B cell follicle formation, reduced perivascular $\mathrm{T}$ cell cuffing, and reduced accumulation of arginase-1-expressing macrophages within the granulomas (Supplemental Figure 4, D-F). As macrophages and not neutrophils are the predominant producers of arginase- 1 in coinfected mice (Supplemental Figure 1, E and F), these results provide direct evidence that accumulation of arginase-1-expressing macrophages in the lungs following S. mansoni infection mediates exacerbated inflammation and disease severity during $M t b$ coinfection, without affecting mycobacterial control.

SEA immunization exacerbates lung inflammation during $M t b$ infection. In addition to the generation of type 2 granulomas, $S$. mansoni coinfection in $M t b$-infected mice may also alter tissue architecture due to the physical presence of the parasite and, thus, exacerbate lung pathology. Therefore, we sought to determine whether exposure to the helminth product $S$. mansoni egg antigen (SEA), which contains the major antigens that induce Th2 responses during infection (15), is by itself sufficient to exacerbate lung inflammation in TB. Thus, C57BL/6 mice were aerosol infected with low doses of $M t b$ and were immunized with SEA at day 15 after infection, a time when $M t b$-specific $\mathrm{T}$ cell responses are initiated in the mouse model of TB (23). We found that mice that were SEA immunized and $M t b$ infected demonstrated increased lung inflammation (Figure 5A), and type 1 granulomas harbored fewer iNOS-expressing macrophages (Figure 5B). In contrast to S. mansoni/Mtb-infected lungs, we did not observe distinct arginase-1-expressing macrophage containing type 2 granulomas in the lungs of SEA-immunized $M t b$-infected mice (data not shown) but nevertheless found increased expression of Arg1 mRNA in sorted lung $\mathrm{CD} 11 \mathrm{c}^{+}$cells from SEA-immunized $M t b$-infected mice (Figure $5 \mathrm{C}$ ). Similarly, we found that the formation of B cell follicles was reduced and less organized in SEA-immunized $M t b$-infected mice (Figure 5D), and this coincided with decreased accumulation of $\mathrm{T}$ cells within lymphoid follicles, which instead accumulated as $\mathrm{T}$ cell perivascular cuffs (Figure $5 \mathrm{E}$ ). Importantly, mice similarly immunized with a control antigen such as BSA or a Th1 promoting antigen such as purified protein derivative (PPD) did not promote lung inflammation, instead limiting inflammation (Supplemental Figure 5, A and B). In addition, mice immunized with either BSA or PPD did not affect Mtb burden (Supplemental Figure 5C). Together, these data suggest that exacerbated lung inflammation and decreased formation of protective $B$ cell follicles can be recapitulated by immunization with SEA in $M t b$-infected mice, suggesting that the inflammatory effects of the coinfection are due to the presence of Schistosoma antigens and likely not due to tissue alterations caused by the physical presence of the helminth parasite. 
A

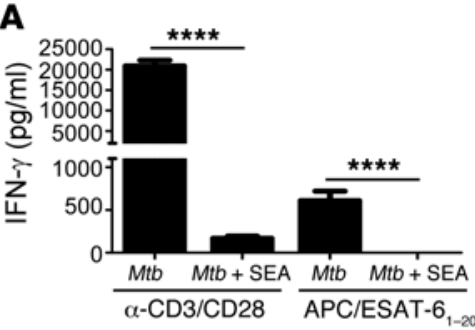

B

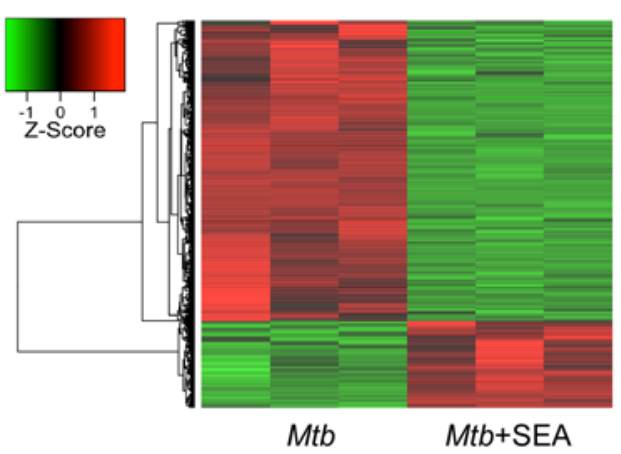

C
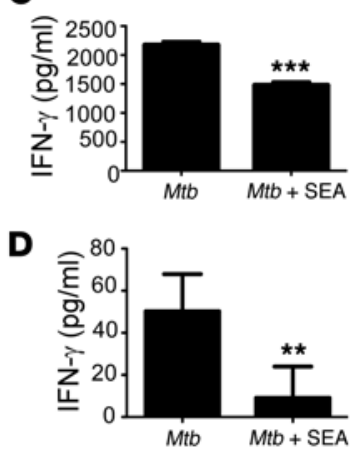
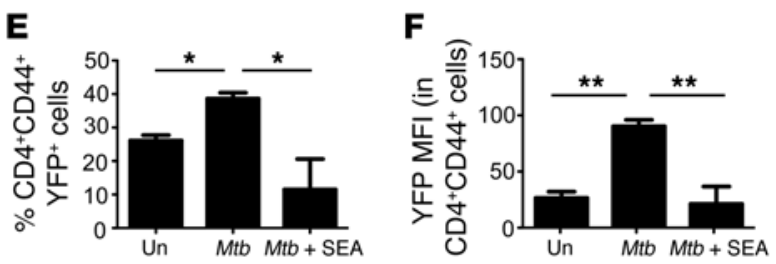

G
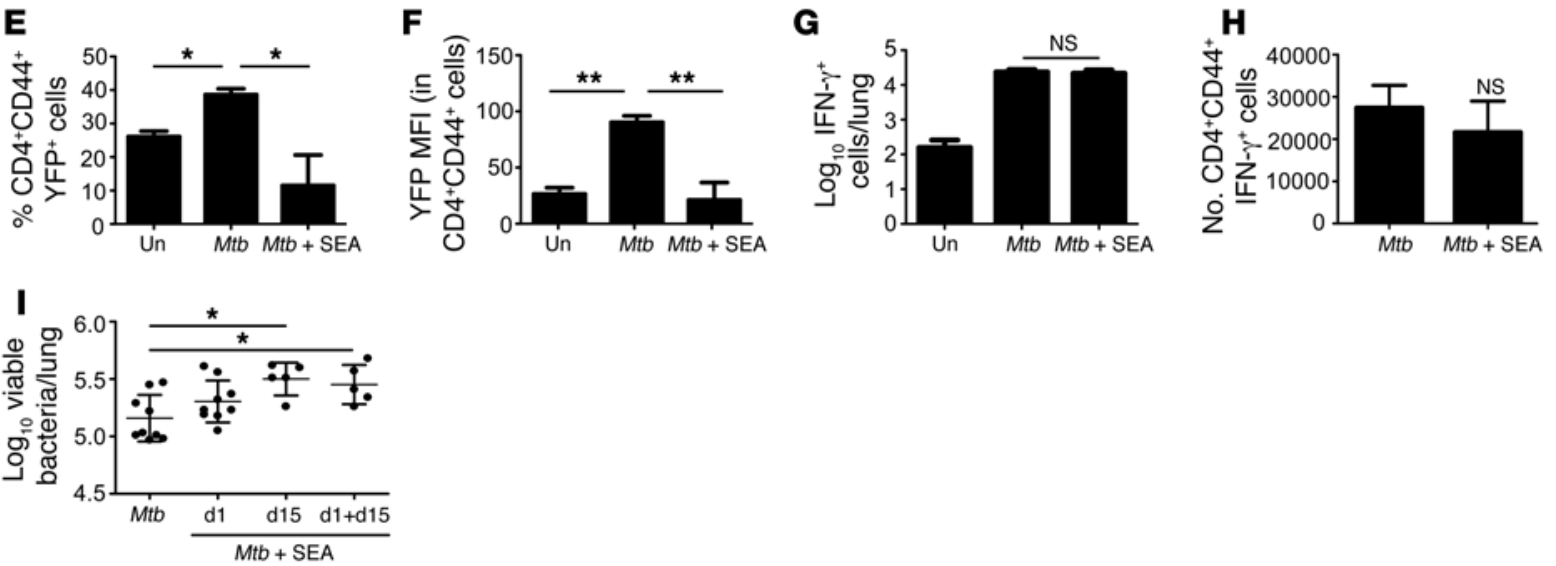

Figure 6. SEA treatment impairs Mtb-driven Th1 responses and increases susceptibility to Mtb infection. (A) Lung CD4 ${ }^{+}$cells were purified from d30 $M t b$-infected mice and incubated with Schistosoma egg antigen (Mtb + SEA) or without SEA (Mtb) and APCs. Cells were restimulated with $\alpha$-CD3/CD28 beads or irradiated splenocytes, and ESAT- $6_{1-20}$ peptide and supernatant IFN- $\gamma$ levels were assessed. (B) Overall gene-expression changes induced by SEA in cell pellets from A were assessed by RNAseq. Heat-map representation of significant genes is shown. (C) CD4+ cells sorted from ESAT-6 TCR Tg mice were transferred to $\mathrm{Cd} 4^{-/-}$mice, which were $\mathrm{Mtb}$ infected. On d30 after infection, lung $\mathrm{CD} 4^{+}$cells were sorted, treated with SEA, and restimulated with irradiated splenocytes and ESAT- $6_{1-20}$ peptide. Supernatant IFN- $\gamma$ levels were determined. (D) In some experiments, Mtb-infected C57BL/6 mice were immunized with SEA or saline on d15 after infection. Lung IFN- $\gamma$ levels from d30 were determined. (E) Mtb-infected Yeti mice were immunized with SEA on d15 after infection, and the percentage of lung CD4+CD44+YFP+ cells was determined by flow cytometry. (F) IFN- $\gamma$ MFI (YFP) within CD4+CD44 ${ }^{+}$T cells was determined. (C) The number of lung ESAT- $6_{1-20}$-specific, IFN- $\gamma$-producing cells was determined by ELISpot. (H) Lung CD4+CD44+IFN- $\gamma^{+}$cell numbers following PMA/ionomycin stimulation were determined. (I) C57BL/6 mice were infected with Mtb and immunized with SEA or saline on d1, d15, or both d1 and d15 after infection, and d30 lung bacterial burden was determined. $n=3$ samples (A and $\mathbf{B}$ ) or $n=5-9$ mice $(\mathbf{C}-1)$. ${ }^{*} P \leq 0.05,{ }^{* *} P \leq 0.01,{ }^{* * *} P \leq 0.001$, ${ }^{* * * *} P \leq 0.0001$; 1-way ANOVA with post-hoc Tukey (A, E, F, G, and I) or unpaired, 2-tailed Student's $t$ test (C, $\mathbf{D}$, and $\left.\mathbf{H}\right)$ were used. One experiment of 2 shown. Un, uninfected controls.

SEA-mediated inhibition of Th1 responses in Mtb infection is reversible. Aerosol infection of mice with $M t b$ results in a chronic pulmonary infection that induces a predominantly Th1 immune response in the lung (23). Consistent with this, we found that $\mathrm{CD} 4^{+} \mathrm{T}$ cells isolated from the lungs of chronically $M t b$-infected WT C57BL/6 mice strongly expressed mRNA for Ifng, Tnf, Stat1, and Il12rb2, which are associated with the Th1 cell pathway (Supplemental Table 1), but not canonical Th2 (Gata3, Il4ra) or Th17 genes (Il17a, Rorc). In vitro treatment of such isolated CD $4^{+} \mathrm{T}$ cells with SEA in the presence of irradiated APCs inhibited both polyclonal (anti-CD3/CD28 bead stimulated) and $M t b$-specific IFN- $\gamma$ production (Figure 6A), without detectable IL-4 production in culture supernatants (data not shown). Upon analysis of gene expression in these cell pellets, we found genes associated with Th1 function (Ifng, Tbx21, and Cxcr3), T cell signaling (Lck, Cd3g, Zap7O, and $L a t)$, T cell costimulation ( $C d 40 \mathrm{lg}, \mathrm{Icos}$, and $C d 28)$, and $\mathrm{T}$ cell activation (Il2ra) were downregulated in SEA-treated cells (Supplemental Table 2 and Figure 6B), while expression of the Th2 cytokines Il4, Il5, and Il13 was below the threshold of detection. To further address if the inhibition of IFN- $\gamma$ production occurs in $M t b$-specific Th cells, we adoptively transferred naive $\mathrm{CD}^{+}{ }^{+} \mathrm{T}$ cells isolated from the $M t b$ early secretory antigenic target 6 (ESAT-6) $\mathrm{T}$ cell receptor (TCR) transgenic (Tg) mice into $C d 4^{-/-}$mice, infected the mice with low doses of aerosolized $M t b$, and isolated in vivo expanded Th cells from the lungs on day 30 after infection. Consistent with our previous data, these isolated Th cells produced IFN- $\gamma$ upon restimulation with ESAT-6, but in vitro SEA treatment in the presence of APCs inhibited the production of IFN- $\gamma$ (Figure 6C) without inducing detectable levels of IL-4 (data not shown).

Next, we assessed whether, similar to Schistosoma infection, SEA immunization affects Th1 immunity in vivo. We found that total IFN- $\gamma$ levels in lung homogenates were reduced in SEAimmunized $M t b$-infected mice when compared with $M t b$-infected lungs (Figure 6D). Next, to assess the effect of SEA at the cellular level, using IFN- $\gamma$ reporter mice (Yeti mice), we found that SEA immunization decreased both the accumulation of activated 
A
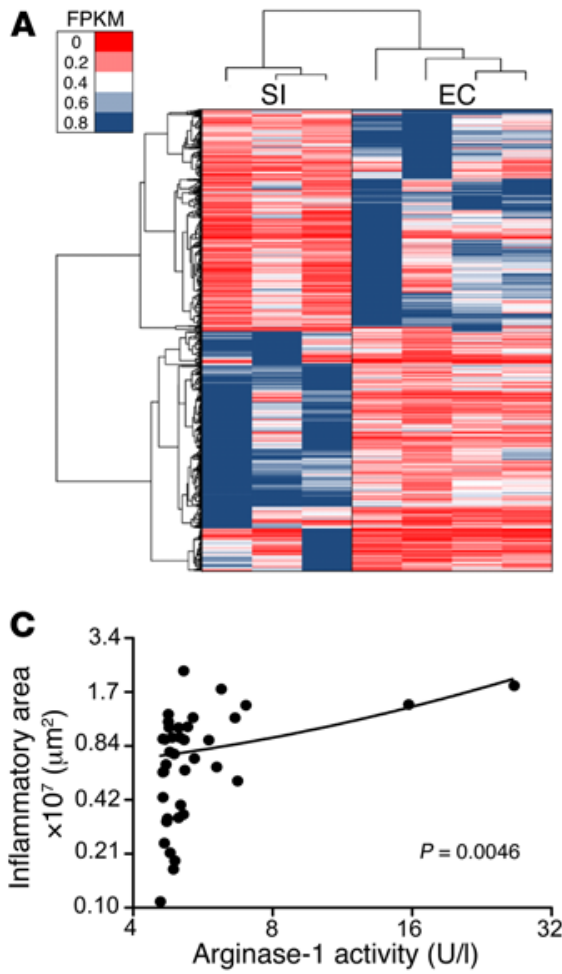

E

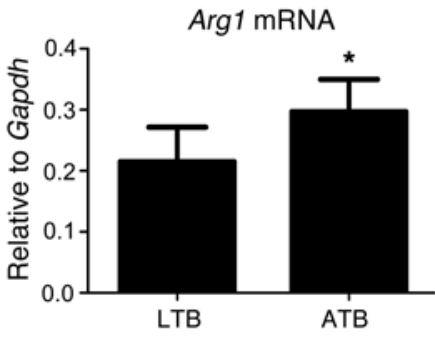

B
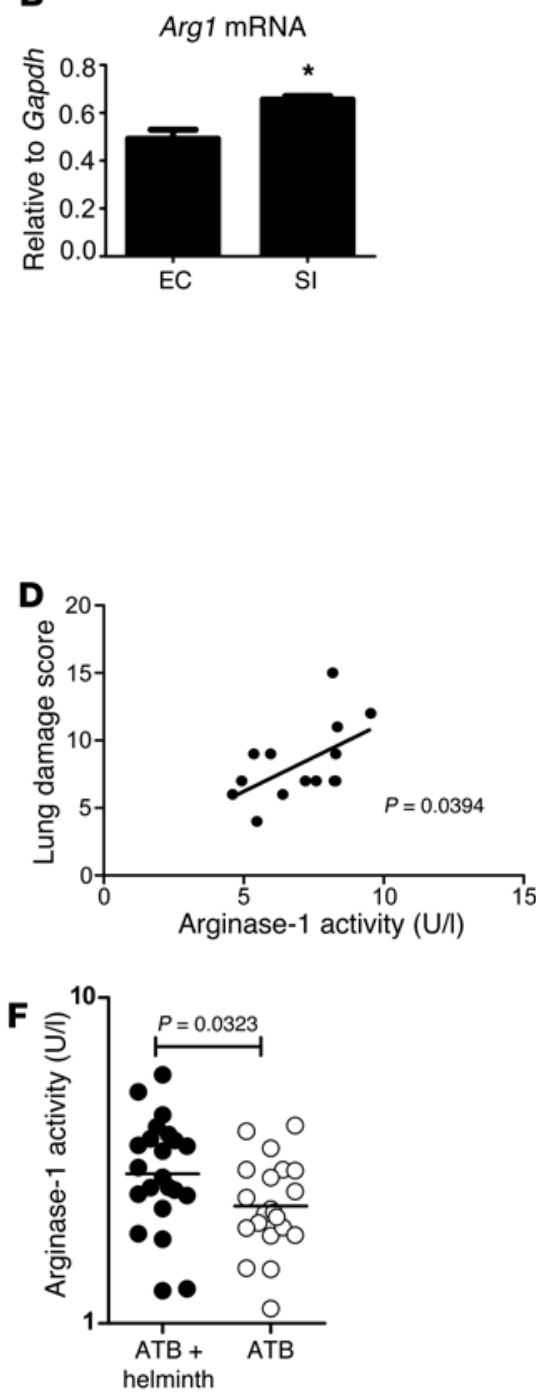

Figure 7. Arginase-1 expression during Mtb infection in genetically diverse mice and in humans correlates with increased inflammation and lung damage. Individual DO $(n=40)$ mice were aerosol infected with $M t b$, and lung bacterial burden on $\mathrm{d} 50$ after infection was determined. Total area occupied by inflammatory lesions per lobe was quantified in H\&E-stained formalin-fixed, paraffin embedded (FFPE) lung sections. RNAseq was performed on lung RNA from individual DO mice with the most SI and individual DO mice with $\mathrm{EC}$, which also correlated with improved formation of lymphoid follicles within the granulomas. (A) Heat-map representation of significant genes. (B) The levels of Arg1 mRNA expression relative to Gapdh in lungs of individual DO mice with SI or EC was determined by RT-PCR. (C) Arginase-1 activity was determined in lung homogenates of individual DO, and linear correlation between arginase-1 activity and lung inflammatory area was determined (Pearson's correlation, $\log _{2}$ scale). (D) Linear correlation analysis of arginase-1 activity in serum of patients with ATB and their lung damage score was carried out (Pearson's correlation, $n=13$ ). (E) The levels of $A R G 1$ mRNA expression relative to $G A P D H$ in blood monocyte cDNA derived from LTB $(n=5)$ and ATB $(n=6)$ human donors were determined. (F) Arginase-1 activity in serum from ATB patients with helminth coinfection and those without helminth coinfections was measured ( $n=22$ each). ${ }^{*} P \leq 0.05$; unpaired, 2-tailed Student's $t$ test (B, E, and $\mathbf{F}$ ) was used.
IFN- $\gamma$-producing $\mathrm{CD}^{+} \mathrm{T}$ cells in $M t b$-infected animals (Figure 6E) and levels of IFN- $\gamma$ reporter expression by activated CD $4^{+}$Th cells in the lungs of $M t b$-infected mice (Figure $6 \mathrm{~F}$ ). Strikingly, assessment of IFN- $\gamma M t b$-specific $\mathrm{T}$ cell responses by restimulation with $M t b$ antigen in ELISpot assays, or following stimulation with PMA/Ionomycin, showed no differences between SEA-treated and $M t b$-infected groups (Figure 6, G and $\mathrm{H}$ ). These data together suggest that SEA-mediated inhibition of IFN- $\gamma$ expression in $\mathrm{CD}^{+}$ $\mathrm{T}$ cells evident in vitro (in cultured Th cells with SEA and APCs) and ex vivo (as assessed by reporter expression) can be reversed by in vitro culture in the absence of SEA (as assessed by ELISpot and intracellular staining). These data suggest that the effects of SEA on Th cell cytokine production and activation profile can be potent in in vitro cultures, but the effects are reversible upon removal of SEA. Additionally, activation of lung macrophages as measured by MHCII expression was reduced in SEA-treated $M t b$-infected mice (data not shown). Incidentally, decreased Th1 responses and decreased macrophage activation coincided with a small but significant increase in bacterial burden in the lungs of SEA-immunized $M t b$-infected mice (Figure 6I). Interestingly, immunization with SEA on day 15 but not day 1 in $M t b$-infected mice coincided with increased susceptibility to TB, suggesting that the presence of SEA during initiation of $M t b$-specific T cell responses, but not earlier, affects protective outcomes during $M t b$ infection (Figure 6I).

In vitro SEA-treated $\mathrm{CD}^{+} \mathrm{T}$ cells from $M t b$-infected lungs that demonstrated impaired $\mathrm{T}$ cell activation and IFN- $\gamma$ production (Figure 6, A and B) - upon subsequent washes to remove SEA and further incubation with $M t b$-infected alveolar macrophages - could now produce IFN- $\gamma$ and activate macrophages to induce iNOS (Supplemental Figure 6, A and B), which coincided with control of $M t b$ infection in infected alveolar macrophages, albeit not to the same level as $\mathrm{CD} 4^{+} \mathrm{T}$ cells isolated from $M t b$-infected lungs (Supplemental Figure 6C). Not surprisingly, naive lung CD $4^{+} \mathrm{T}$ cells isolated from uninfected mice, which demonstrated poor induction of IFN- $\gamma$ production in T cells (Supplemental Figure 6A) and decreased induction of iNOS (Supplemental Figure 6B), exhibited reduced $M t b$ control in alveolar macrophages (Supplemental Figure 6C). These data together with the improved $M t b$ control observed in praziquantel-treated coinfected mice (Figure $3 G$ ) indicate that, although the presence of helminth antigens is sufficient to dampen $M t b$-specific Th1 responses, these effects are reversible upon removal of the parasite and/or its products. 
Helminth infections are associated with increased TB reactivation rates in HIV-infected patients (10). Thus, based on our data that $S$. mansoni infection or immunization with SEA can exacerbate lung inflammation and increase $M t b$ susceptibility, we then tested whether SEA immunization would affect disease progression in mice undergoing TB reactivation. Using the Cornell model of $M t b$ reactivation (24), chronically $M t b$-infected mice were treated with isoniazid and rifampicin until no cultivable lung bacteria were recovered (14), and mice were immunized with SEA and spontaneously allowed to reactivate $M t b$ infection. We found that SEAimmunized mice had higher lung and spleen bacterial burden when compared with control mice that also reactivated $M t b$ infection (Supplemental Figure 7). These data, for the first time to our knowledge, provide experimental evidence that TB reactivation can be accelerated due to the presence of helminth products such as SEA, likely due to its effect on both Th1 responses and due to decreased formation of lymphoid follicle-containing lung granulomas.

Arginase-1 expression is associated with exacerbated inflammation in TB. Our data demonstrate that arginase-1 induced in response to $S$. mansoni infection and SEA immunization exacerbates inflammation in inbred C57BL/6 Mtb-infected mice. Inbred mice do not exhibit the heterogeneity in inflammatory responses seen in human TB. Thus, we recently used the diversity outbred (DO) mouse population to model heterogeneity in lung inflammatory and protective outcomes upon $M t b$ infection (14). We next addressed if arginase- 1 expression in $M t b$-infected DO hosts, even in the absence of helminth coinfection, was associated with exacerbated lung inflammation. Thus, we carried out RNA sequencing on lungs of individual genetically diverse DO mice that had severe inflammation (SI) and compared their transcriptional expression profile to individual DO mice that had enhanced control (EC), which coincided with formation of lymphoid follicle-containing granulomas and decreased $M t b$ burden (14). Interestingly, we found that Arg1 mRNA was among the top 10 genes differentially expressed in mice with SI, when compared with genes expressed in lungs of mice with enhanced $M t b$ control (Supplemental Table 3 and Figure 7A). In support of recently published studies $(14,25)$, mRNA for inflammatory molecules such as S100a8 and S100a9, and chemokines associated with neutrophilic accumulation, were also increased in the lungs of mice with SI at the mRNA level (Supplemental Table 3). The increased expression of Arg1 mRNA was confirmed by RT-PCR (Figure 7B). Importantly, arginase-1 activity in lung homogenates of individual DO mice correlated with increased pulmonary inflammation (Figure 7C). Thus, we then addressed whether arginase- 1 could be an inflammatory marker that correlates with the extent of lung damage in human ATB patients in a TB endemic setting. We found that levels of arginase- 1 activity in the serum from ATB patients showed a positive linear correlation with the extent of lung inflammatory damage as assessed by chest radiographs (Figure 7D). This also coincided with increased relative expression of $A R G 1$ mRNA in CD14 ${ }^{+}$ monocytes isolated from peripheral blood of ATB patients when compared with LTB patients (Figure 7E). Importantly, we also found increased serum arginase- 1 activity in ATB patients with helminth coinfections when compared with ATB patients without helminth coinfections (Figure $7 F$ ). These data provide new evidence that, despite the well-known role for arginase- 1 in impairing
Th1 responses, arginase-1 may have a new emerging role to play in mediating pulmonary inflammation in TB.

\section{Discussion}

TB remains one of the largest global health threats, and it is estimated that $5 \%-10 \%$ of infected individuals are likely to progress to ATB. However, the mechanisms leading to TB reactivation in otherwise immunocompetent individuals are largely undefined. As such, coinfection with helminths is considered a risk factor associated with increased TB reactivation rates and increased susceptibility to TB. However, thus far, the mechanisms behind this association remain poorly defined. In this study, we demonstrate that coinfection with $S$. mansoni, or presence of $S$. mansoni products such as SEA, can reversibly impair Th1 immune responses, but its effects on $M t b$ control are minimal. Unexpectedly, our data demonstrate that the more prominent effect of helminth infection during TB is the accumulation of high arginase-1expressing macrophages within type 2 granulomas in the lung, mediating exacerbated inflammation and lung pathology during coinfection. Importantly, the effects driven by helminth-induced arginase-1-expressing macrophages on inflammation are reversible, as treatment with antihelminthics can improve TB disease outcome. Our data in genetically diverse DO mice infected with $M t b$ and humans with ATB also show that increased arginase- 1 activity correlates with increased inflammatory damage and disease severity during TB. Furthermore, helminth coinfection in ATB patients increases arginase- 1 activity in serum. Together, our data suggest that, contrary to the well-known antiinflammatory role for arginase- 1 and its inhibitory effects on Th1 responses (26), arginase-1 has a new prominent role in driving inflammation and mediating lung damage during TB.

Evidence suggests that a skewed Th2 response caused due to $S$. mansoni infection compromises protection conferred by $M$. bovis BCG vaccination in humans (7-9) and in mice systemically infected with $M t b$ (12). Similarly, other models of helminth coinfections such as Nippostrongylus brasiliensis, a rodent intestinal helminth that migrates to the lung, also drives Th2 responses and generation of alternatively activated macrophages in the lung, resulting in impaired resistance to $M t b$ (27). Interestingly, while helminth coinfections increase $M$. bovis BCG (11) and Mtb (27) lung burden, the increase in mycobacterial burden is often transient (27) and moderate (about 1 log higher in coinfected hosts) $(11,27)$, suggesting that the effect of helminth coinfections on $M t b$ control is not profound enough to fully explain the increased severity of TB in helminth-coinfected individuals observed in TB endemic regions. Furthermore, in some models of helminth/Mtb coinfection, there are no differences observed in overall mycobacterial control $(28,29)$. Our results support these published studies, as $S$. mansoni coinfection or immunization with SEA resulted in increased $M t b$ burden in the lung. However, this effect was not profound, suggesting that $M t b$ control mechanisms are not completely suppressed in coinfected hosts. Furthermore, while some evidence suggests that $M t b$-specific Th1 responses are impaired due to helminth coinfection $(7,11,30)$, other studies have found that $M t b$-specific Th1 responses are unaltered in the presence of helminth infections $(27,29)$. Our studies show that $M t b$ infection induces a potent Th1 signature in in vivo primed $\mathrm{T}$ cells isolated 
from the infected lung and that in vitro treatment with SEA has an inhibitory effect on Th1 immune responses, as well as on cell activation pathways. Similarly, the presence of SEA or S. mansoni during $M t b$ infection in vivo results in a significant inhibition of IFN- $\gamma$ production in T cells (Figure $2 \mathrm{E}$ ). However, we demonstrate that these effects on cytokine production by Th1 cells are transient and reversible, as evidenced by their comparable ability to produce IFN- $\gamma$ following in vitro restimulation. In addition, removal of SEA restores IFN- $\gamma$ production and the ability to activate alveolar macrophages to control $M t b$ infection. The discrepancy between our ex vivo flow cytometric analysis using reporter mice and our ELISpot results highlights the importance of directly analyzing immune cell function in the absence of additional restimulation in vitro. The differential assays used in the various studies may in part be responsible for the variability in the impairment of $M t b$-specific Th1 responses observed due to helminth infections across studies $(7,11,27,30)$. Nonetheless, it is quite surprising that, despite impairment of Th1 responses by treatment with SEA in vitro, removal of SEA in culture conditions can reverse this impairment. This is further supported by our data that show that treatment of $S$. mansoni infection with an antihelminthic such as praziquantel can reverse the accumulation of arginase-1-expressing lung macrophages and decrease disease severity during TB. These findings are notable and underscore the potential for the broad application of antihelminthics as a cost-effective strategy to overcome the effects of helminth-driven arginase-1-mediated disease severity during TB and to decrease the risk for TB reactivation in susceptible, coinfected individuals.

Our data demonstrate that, while the effects of Schistosoma coinfection on $M t b$ control are small but significant, its influence on $M t b$-induced lung inflammation and pathology are dramatic. Considering that the $M t b$ burden was not profoundly different in coinfected mice when compared with $M t b$-infected mice, the exacerbated inflammation observed in coinfected hosts is likely not due to differences in the mycobacterial antigenic load. Instead, we demonstrate that helminth infection results in induction of high arginase-1-expressing macrophages within the lung, which are organized as type 2 granulomas and are morphologically and functionally different from the type 1 granulomas containing high iNOS-expressing macrophages induced in response to $M t b$ infection. The induction of high arginase-1-expressing macrophages results in increased lung pathology in the S. mansoni/Mtb-coinfected hosts, projecting an inflammatory role for arginase-1 in $M t b$ infection. These data are further supported by the increased expression of Arg1 mRNA expression in CD11c $\mathrm{c}^{+}$lung cells and increased inflammation observed in SEA-treated $M t b$-infected mice, suggesting that the presence of Schistosoma antigens is sufficient to induce inflammation and lung pathology during $M t b$ infection. Our recent studies showed that the presence of B cell follicles harboring $\mathrm{T}$ cells interacting with macrophages within granulomas are associated with $M t b$ control during TB latency (13), while the presence of granulocytes within the tubercle granulomas causes increased inflammation and exacerbates disease severity in clinical disease (14). Accordingly, the increased inflammation associated with Schistosoma coinfection and SEA immunization results in reduced formation of protective lymphoid follicles, with the concomitant formation of inflammatory granulomas containing both neutrophils and eosinophils. These data suggest that accumulation of helminth infection-induced arginase-1-expressing macrophages in the lungs initiates the formation of inflammatory rather than protective granulomas during TB. The fact that S. mansoni/Mtb-coinfected BMC mice that lack arginase-1-expressing macrophages exhibit decreased lung inflammation and increased formation of protective $B$ cell follicles provides direct evidence that helminth-induced arginase- 1 expression in myeloid cells mediates disease exacerbation in coinfected hosts. Interestingly, in a dermal $M t b$-infection model resulting in hypoxic lung granulomas, absence of arginase-1 in macrophages resulted in exacerbated inflammation in iNOS-deficient mice (21). It is possible that arginase-1 expression in macrophages can function to either limit or exacerbate inflammation, depending upon the availability or absence of iNOS, and this needs to be carefully examined in the future. In the context of pulmonary $M t b$ infection, macrophages were found to express either iNOS or ariginase-1, or coexpress both iNOS and arginase-1, with the arginase-1-expressing macrophages localized to lymphocytic cuffs of granulomas in $M t b$-infected macaques (31). Accordingly, $M t b$ infection can induce arginase-1 expression in macrophages (18), and high levels of both arginase-1 and iNOS activity was detected in $M t b$-infected macaque granulomas (31). Consistent with these studies, the correlation between increased arginase- 1 expression and increased inflammation in genetically diverse outbred mice and humans with ATB in our study further suggests that genetic factors that drive increased arginase-1 production may contribute to increased pathology and disease severity during TB. Importantly, similar to the mouse model of coinfection, we detected increased arginase-1 activity in the serum of ATB patients coinfected with helminths when compared with ATB patients without helminth coinfections. This is consistent with the presence of arginase-1-expressing macrophages within granulomas found within lungs of TB patients $(31,32)$, where arginase- 1 is expressed both in granuloma-associated macrophages $(31,32)$ and in type II pneumocytes (32). Arginine is a crucial amino acid that modulates immune responses to $M t b$, as it is a common substrate for both iNOS and arginase-1. Loss of arginase-1 in macrophages enhances protection against $M t b$, possibly due to increased availability of arginine for iNOS activity (18). Interestingly, $M t b$ infection in mice deficient in arginase-1 expression within macrophages also exhibits smaller, more lymphocytic granulomas (18), further supporting our hypothesis that arginase-1 regulates inflammation within TB granulomas. Based on these data, we propose that arginase-1 induced due to helminth coinfections, helminthic products, and host genetics mediates the formation of more inflammatory TB granulomas. Finally, increased reactivation of $M t b$ infection after SEA treatment suggests that helminth coinfection likely facilitates increased TB reactivation rates.

Arginase- 1 has been implicated in models of lung injury, where increased expression of arginase- 1 coincides with collagen deposition and fibrosis in pulmonary diseases such as asthma, cystic fibrosis, idiopathic pulmonary fibrosis (IPF), and chronic obstructive pulmonary disease (COPD) (33). In animal models of silicosis, another inflammatory lung disease characterized by fibrosis, arginase activity and arginase-1 expression are enhanced in alveolar macrophages (33). In contrast, in S. mansoni-induced Th2-driven inflammation, macrophage-specific arginase- 1 was demonstrated 
to function as a suppressor of fibrosis (19). Our studies demonstrate that, in the lungs of mice coinfected with Schistosoma and $M t b$, increased accumulation of arginase-1-expressing macrophages organized as type 2 granulomas results in increased collagen expression and increased Mmp13 and Mip1a mRNA expression (data not shown), which are molecules implicated in lung fibrosis (33). The fact that accumulation of arginase-1-expressing macrophages coincides with exacerbated inflammation in a Th1-dominated disease such as TB is unexpected, as other models of acute and chronic Th2-mediated lung inflammation showed neither a pathogenic nor a protective role for myeloid-expressed arginase-1 (20). In addition, our data show that praziquantel treatment in coinfected mice is able to control $S$. mansoni infection to fully reverse the increased inflammation and higher $M t b$ bacterial burden seen in coinfected mice to levels seen in $M t b$-infected mice. This is in contrast with the coinfection in BMC mice specifically lacking arginase-1 expression in macrophages, where increased inflammation is reversed but increased $M t b$ burden is unaffected. In our studies, we have used the Tie2-Cre Arg1 $1^{f / f l}$ mice to delete arginase-1 expression in all myeloid cells, and the Lysm-Cre Arg $1^{f / / f l}$ mice to delete arginase- 1 specifically in macrophages and neutrophils. Consistent with our data that macrophages are the primary arginase-1-producing cells in the coinfected lung, generation of BMC mice using BM cells from either Tie2-Cre Arg $1^{f / f l}$ mice or Lysm-Cre Arg1 $1^{f / f l}$ mice ablated the accumulation of lung macrophages producing arginase- 1 in coinfected mice and reversed lung inflammation. These data together suggest that, while the increased inflammation seen in coinfected mice is helminth driven and arginase-1 dependent, the increase in $M t b$ burden - although helminth driven - is not directly dependent on arginase-1 expression in macrophages. Future studies delineating the specific mechanisms by which helminth-induced responses drive the fibrosis and increased inflammation in a Th1 disease such as TB will shed further light on the mechanisms of $M t b$ control, disease, and TB reactivation in helminth/TB-coinfected populations.

In conclusion, our data suggest that, contrary to the wellknown antiinflammatory role for arginase- 1 and its inhibitory effects on Th1 responses, arginase- 1 has a prominent role in driving inflammation and mediating lung damage during TB. These data provide new insights into the mechanisms by which helminth coinfections drive increased susceptibility and disease progression in TB. In addition, our data show that the effects of helminth products on impairment of Th1 responses are reversible and support the use of antihelminthics as a cost-effective strategy to improve $M$. bovis BCG vaccination, boost efficient immune responses against $M t b$, and decrease the risk for $\mathrm{TB}$ reactivation in susceptible, coinfected individuals.

\section{Methods}

Human tissue samples and patient diagnosis. Fresh blood samples from ATB patients were obtained from patients recruited to the Tuberculosis Outpatient Clinic (INER, Mexico City, Mexico, or NIH-NIRTICERT). Serum samples from all patients with ATB were collected prior to anti-Mtb treatment and did not present comorbidities such as diabetes, HIV, cancer, and COPD. For ATB patients, chest radiographs were assessed for the presence, distribution, and extent of pulmonary abnormalities, such as airway consolidation and fibrosis, lung distor- tion, traction bronchiectasis, irregular interfaces, and parenchymal bands. We developed a quantitative scale for measuring the degree of lung damage, according to the profusion of pulmonary abnormalities. The pulmonary parenchyma was evaluated in 4 quadrants, with the division between the upper and lower lung in both sides being arbitrarily set at the carina section. Each quadrant was scored from 0-5, where 0 denoted absence of lesions and 5 was the maximum affection. The score represented the percentage of lung parenchyma involvement. The maximum score for the 4 lung zones was 20 (14). The images were blinded, and the same image was read twice separately by an experienced observer (pulmonologist researcher). The reported score is the mean of 2 measurements.

Buffy coats from $20 \mathrm{ml}$ of EDTA anticoagulated peripheral blood from ATB and LTB patients who signed an informed consent letter were obtained at the TB clinic of the INER. Total peripheral blood mononuclear cells (PBMCs) were obtained by density gradient centrifugation using Lymphoprep (Axis-Shield). CD14 ${ }^{+}$monocytes were purified using magnetic beads (Miltenyi Biotec). Purity of isolated monocytes was assessed by flow cytometry using anti-human monoclonal antibodies: CD14-FITC and CD3-PE-Cy7 (BioLegend), obtaining >97\% purity. Total RNA was isolated from monocytes for quantitative PCR (qPCR) expression assays using validated TaqMan assays (ThermoFisher).

In samples collected from NIH-NIRT-ICER, we studied a group of 44 individuals with active pulmonary TB, 22 of whom were infected with the helminth Strongyloides stercoralis. Active pulmonary TB was diagnosed microbiologically on the basis of being at least culture positive for $M t b$ by solid cultures in Lowenstein Jensen medium; some were also sputum smear positive. Strongyloides infection was diagnosed by the presence of IgG antibodies to the 31-kDa recombinant NIE antigen by the luciferase immunoprecipitation system assay, as described previously (34).

Animals. C57BL/6 mice were purchased from Taconic, and IFN- $\gamma$ YFP reporter mice were obtained from Markus Mohrs (Trudeau Institute, Saranac Lake, New York, USA) (35). ESAT-6 Tg mice, expressing the $\alpha \beta$ TCR specific for the IAb-presented ESAT- $6_{1-20}$ peptide, were provided by G. Winslow (Wadsworth Center, Albany, New York, USA) and D. Woodland (Trudeau Institute) (36). The ESAT-6 TCR Tg mice were maintained on the Rag1/-- background. Yarg, Cd4 ${ }^{-/-}$, C57BL/6.SJL, and DO mice were purchased from The Jackson Laboratory. Tie2-Cre Arg $1^{f / f l}$, Lysm-CreArg1 $1^{f / f l} \mathrm{BM}$, and $\operatorname{Arg} \mathrm{f}^{\ell / / l}$ (WT) mice were used as before (18). Mice strains were bred and maintained in house either at the University of Pittsburgh or Washington University in St. Louis. Experimental mice were age- and sex-matched and used between the ages of 6-8 weeks in accordance with University of Pittsburgh and Washington University in St. Louis Animal Care and Use Committee guidelines.

$M t b$ aerosol infection, S. mansoni infection, and immunizations. Mtb strain H37Rv was cultured in Proskauer Beck medium containing $0.05 \%$ Tween 80 to mid-log phase and was frozen in $1 \mathrm{ml}$ aliquots at $-80^{\circ} \mathrm{C}$. For $M t b$ infections, animals were aerosol infected with approximately 100 CFU of bacteria using a Glas-Col airborne infection system. At given time points, organs were harvested and homogenized, and serial dilutions of tissue homogenates were plated on 7H11 agar plates supplemented with oleic albumin dextrose catalase (OADC). Additionally, lung samples were processed for RNA extraction, histology, and cytokine determination. For $S$. mansoni/Mtb coinfections, mice were infected by percutaneous exposure to $55 \mathrm{~S}$. mansoni cercariae (Puerto Rican/Naval Medical Research Institute strain) 6 weeks prior to $M t b$ 
aerosol challenge. S. mansoni liver egg burden was determined by overnight digestion of the liver in $4 \% \mathrm{KOH}$ at $37^{\circ} \mathrm{C}$. Eggs were enumerated by microscopic observation of the liver suspensions. SEA was prepared as described before (37). For immunizations, $50 \mu \mathrm{g}$ of SEA, BSA, or PPD were s.c. administered to mice as described before (37).

Antihelminthic treatment. S. mansoni treatment was performed by oral gavage with $6.25 \mathrm{mg}$ of praziquantel in a volume of $300 \mu \mathrm{l}(38)$. $S$. mansoni/Mtb-coinfected mice received 3 doses of praziquantel 24, 72, and 120 hours following $M t b$ infection. Control mice instead received $300 \mu \mathrm{l}$ of PBS.

Generation of BMC mice. To generate BMC mice, we treated C57BL/6.SJL mice with Sulfamethoxazole and Trimethroprim (Hi-Tech Pharmacal) in the drinking water for 2 weeks prior to irradiation. Mice were sublethally irradiated with 1,000 rad and were reconstituted with $5 \times 10^{6}$ to $10 \times 10^{6} \mathrm{BM}$ cells from either WT control $\left(\mathrm{Arg} 1^{f / f t}\right)$ or Tie2-Cre Arg1 $1^{l / f l}$ mice or Lysm-Cre Arg1 $1^{l / f l}$ via i.v. injection as before (13). Mice were allowed to reconstitute for 6 weeks while continuing to receive antibiotic water, after which they were used in experimental procedures. BM reconstitution was verified by flow cytometric analysis of peripheral blood cells.

Modified Cornell mouse model of LTB. C57BL/6 mice were aerosol infected with approximately $100 \mathrm{CFU}$ of $\mathrm{Mtb} \mathrm{H} 37 \mathrm{Rv}$ using a Glas-Col airborne infection system and rested for 30 days. Mice were subsequently treated for 6 weeks with rifampicin $(100 \mathrm{mg} / \mathrm{l})$ and isoniazid $(200 \mathrm{mg} / \mathrm{l})$ in the drinking water (14). After a 2-week period, mice received either 2 s.c. immunizations of $50 \mu \mathrm{g}$ SEA or PBS. Lung and spleen bacterial burden was determined 2 weeks after the second immunization, as described above.

$C D 4^{+} T$ cell isolation, in vitro $T$ cell differentiation and restimulation. Single cell suspensions from DNAse/collagenase-treated lung tissue or spleen were prepared as previously described (39). CD4 ${ }^{+} \mathrm{T}$ cells from secondary lymphoid organs of ESAT- 6 TCR Tg mice or from the lungs of $M t b$-infected mice were isolated using magnetic $\mathrm{CD} 4^{+}$beads (clone GK1.5, Miltenyi Biotec), according to the manufacturer's instructions. For SEA treatment, cells were incubated in the presence of irradiated splenocytes, $5 \mu \mathrm{g} / \mathrm{ml}$ ESAT- 6 peptide, $10 \mathrm{U} / \mathrm{ml} \mathrm{IL-2} \mathrm{with} 50 \mu \mathrm{g} / \mathrm{ml} \mathrm{SEA}$. $\mathrm{T}$ cells were incubated for 6 days at $37^{\circ} \mathrm{C}$ and $5 \% \mathrm{CO}_{2}$ and supplemented with an equivalent volume of media containing IL-2 (10 U/ml) on day 3. Control cells were incubated using the same peptide and cytokine concentrations in the absence of SEA. After 6 days, T cells were restimulated with irradiated splenocytes and $5 \mu \mathrm{g} / \mathrm{ml}$ ESAT- 6 peptide for 48 hours. Tissue culture supernatants were then harvested and used in ELISA, while cell pellets were processed for RNA extraction.

Arginase-1 activity determination. Arginase-1 activity in serum or lung homogenates was determined using the QuantiChrom Arginase Assay Kit (BioAssay Systems). For serum samples, urea was eliminated by performing 2 washes with PBS through Amicon Ultra 0.5 (10 kDa cutoff, Millipore). For lung homogenates, protein concentration was adjusted to $100 \mu \mathrm{g} / \mathrm{ml}$. Sample $(40 \mu \mathrm{l})$ was incubated at $37^{\circ} \mathrm{C}$ for 120 minutes in the presence or absence of $10 \mu \mathrm{l}$ of $5 \times$ assay reagent. Urea levels were subsequently detected and calculated according to the manufacturer's instructions. Arginase- 1 activity is expressed as U/l of sample.

Cytokine and chemokine protein determination. Culture supernatants or lung homogenates were generated, and cytokine and chemokine concentrations were determined by sandwich ELISA (Mouse DuoSet ELISA, R\&D Systems) or Luminex (Millipore), according to the manufacturer's instructions.
Flow cytometry. Single cell lung suspensions from lung tissue were stained with fluorochrome-labeled antibodies specific for CD3 (clone 145-2C11), CD4 (clone RM4-5), CD44 (clone IM7), CD11c (clone HL3), CD11b (clone M1/70), MHCII (clone M5/114.15.2), CD49B (clone DX5), FceRI (clone MAR-1), F4/80 (clone BM8), Siglec-F (clone E50-2440), CD8a (clone 53-6.7), CD117 (clone 2B8), Gr-1 (clone RB6$8 \mathrm{C} 5$ ), or isotype control antibodies (BD Biosciences). For intracellular cytokine staining, lung cell suspensions were stimulated with $50 \mathrm{ng} /$ ml PMA (Sigma-Aldrich), $750 \mathrm{ng} / \mathrm{ml}$ ionomycin (Sigma-Aldrich), and GolgiStop (BD Biosciences) for 5 hours at $37^{\circ} \mathrm{C}$. Following Fc block incubation (BD Biosciences), surface staining was carried out. Cells were then permeabilized with Cytofix-Cytoperm solution (BD Biosciences) and stained with anti-IFN- $\gamma$ (XMG1.2) or anti-IL-4 (11B11) (BD Biosciences). Cells were analyzed using a BD LSR II flow cytometer using FACS Diva software (BD Biosciences). Cells were gated based on their forward by side scatter characteristics, and the frequency of specific cell types was calculated using FlowJo. Mean fluorescence intensity was also determined to define expression levels of different molecules using FlowJo. Lung macrophages and neutrophils were sorted from lung single cell suspensions prepared from coinfected mice on a FACsJazz (BD Biosciences) instrument using fluorochrome-labeled antibodies. Lung macrophages were identified as $\mathrm{F} 4 / 80^{+} \mathrm{CD} 11 \mathrm{c}^{+}$cells, while neutrophils were identified as $\mathrm{Gr}^{+} / \mathrm{CD} 11 \mathrm{~b}^{+}$cells, with resulting purity of $>95 \%$ for sorted macrophages and $>85 \%$ for neutrophils.

Real-time PCR. RNA was extracted using the RNeasy kit (QIAGEN), and CDNA was then amplified with FAM-tagged probes and PCR primers on a Viia7 thermocycler. Specific gene expression was calculated relative to Gapdh mRNA expression. Primer and probe sequences targeting specific genes were commercially purchased (ABI Biosystems).

Detection of IFN- $\gamma$-producing cells by ELISpot. ESAT- $6_{1-20}$-specific IFN- $\gamma$-producing $\mathrm{CD}^{+} \mathrm{T}$ cells from infected lungs were quantified using peptide-driven ELISpot. Briefly, 96-well ELISpot plates were coated with monoclonal anti-mouse IFN- $\gamma$ (clone R46A2, eBioscience) in phosphate-buffered saline and blocked with media supplemented with $10 \%$ FBS. Cells from lungs were plated at an initial concentration of $5 \times 10^{5}$ cells/well, and serial 2-fold dilutions were made. Restimulation was carried out in the presence of $1 \times 10^{6}$ cells/well C57BL/6 irradiated splenocytes, ESAT- $6_{1-20}$ peptide $(10 \mu \mathrm{g} / \mathrm{ml})$, and mouse rIL-2 (10 U/ml). After 24 hours, plates were washed and probed with biotinylated anti-mouse IFN- $\gamma$ (XMG1.2, eBioscience). Spots were enumerated using a CTL-immunospot S5 MicroAnalyzer (Cellular Technology Limited). No spots were detected in cultures lacking antigen.

RNA seq. Total RNA was isolated using the RNEasy Mini kit (QIAGEN), and RNA integrity was determined with the Agilent 2100 Bioanalyzer. Briefly, mRNA was purified from 2-5 $\mu$ g of total RNA using Sera-Mag Oligo(dT) Beads, fragmented with magnesiumcatalyzed hydrolysis, and reverse transcribed into cDNA using random primers (Superscript II; Invitrogen). Then, cDNA underwent end repair with T4 DNA polymerase and Klenow DNA polymerase, followed by the addition of 'A' bases to the 3 ' end and ligation to adaptor oligos. Products from the ligation were run on a $2 \%$ agarose gel. A gel slice consisting of the $200 \mathrm{bp}$ region ( $\pm 25 \mathrm{bp}$ ) was excised and used as a template for PCR amplification. The final PCR product was purified, denatured with $2 \mathrm{~N} \mathrm{NaOH}$, and diluted to $10-12 \mathrm{pM}$ prior to cluster amplification on a single-read flow cell v4, as outlined in the Single-Read Cluster Generation Kit v4 (Illumina). The flow cell was sequenced on an Illumina Genome Analyzer II. 
We mapped reads to Mus musculus (mm9) reference transcriptome from GenBank using the Burrows-Wheeler aligner tool. For sample-wise data normalization, we applied DESeq using R statistical software. To achieve more reliable results, we applied 3 popular methods for detecting differentially expressed genes (DEGs) using RNAseq data: edger, DESeq, and baySeq. We considered genes detected by at least 2 methods as DEGs when they had a q-value (FDR) less than 0.05. Pathway analysis was performed using Ingenuity IPA. $P$ values from Fisher's exact test were adjusted by Benjamini-Hochberg multiple testing correction. All data sets have been deposited in NCBI with accession numbers GSE66578 and SRX960261-267.

Morphometric analysis and immunofluorescence. Lungs from $M t b$-infected mice were inflated with $10 \%$ neutral buffered formalin and were paraffin embedded. Lung sections were stained with H\&E stain (Colorado Histo-Prep) and processed for light microscopy. To visualize collagen deposition, Gomori's trichrome staining (SigmaAldrich) was performed according to the manufacturer's instructions. Mucus was detected by PAS stain. Slides were prepared for immunofluorescent staining by deparaffinization and subsequent rehydration by washes with xylene, alcohol, and PBS. Antigen retrieval was performed using a Dako Target Retrieval Solution, followed by blocking with $5 \%$ (v/v) normal donkey serum and Fc block $(5 \mu \mathrm{g} / \mathrm{ml}$, 2.4G27). Endogenous biotin was neutralized with avidin, followed by biotin (Sigma-Aldrich). Sections were stained with goat anti-mouse CD3ع (M-20; Santa Cruz Biotechnology Inc.), rat anti-mouse B220 (RA3-6B2; BD Biosciences), inducible NO synthase (goat anti-mouse [M-19; Santa Cruz Biotechnology Inc.]), F4/80 (MCA497GA, AbD Serotec), arginase-1 (rabbit anti-arginase I [H-52; Santa Cruz Biotechnology Inc.]), and biotin monoclonal mouse anti-mouse Muc5AC (45M1; Abcam). Primary antibodies were detected with secondary antibodies conjugated to Alexa fluor 568 for iNOS and CD3 (A-11057; Invitrogen). Donkey anti-rat $\mathrm{Ab}$ conjugated to Alexa Fluor 488 was used to visualize B220 (A-21208; Invitrogen), donkey anti-rabbit conjugated to FITC was used to visualize arginase-1 (711-095-152; Jackson ImmunoResearch Laboratories Inc.). Streptavidin-Alexa Fluor 488 was used to visualize Muc5AC (Invitrogen). Slow fade gold antifade with DAPI (Invitrogen) was used to counterstain tissues and to detect nuclei. Images were obtained with a Zeiss Axioplan 2 microscope and were recorded with a Zeiss AxioCam digital camera. Caudal lobes underwent morphometric analysis in a blinded manner using the morphometric tool of Zeiss Axioplan microscope, which determines the area defined by the squared pixel value for each granuloma and B cell follicle (13).

In vitro Mtb-killing assay. Bronchoalveolar lavage was performed on euthanized naive C57BL/6 mice with sterile PBS containing 0.2 mM EDTA. Following centrifugation, cells were incubated for 1 hour at $37^{\circ} \mathrm{C}$, after which adherent cells were used for subsequent studies. $\mathrm{CD}^{+} \mathrm{T}$ cells from $M t b$-infected mice were sorted as previously described and treated in the presence or absence of SEA for 6 days. Following restimulation, $\mathrm{T}$ cells were washed extensively and then layered over $M t b$-infected alveolar macrophages (MOI 1:1) for 6 days. At the end of the culture period, macrophages were washed twice with PBS and lysed by a 5 -minute incubation with $0.05 \%$ SDS. Following SDS neutralization with $10 \%$ BSA, intracellular $M t b$ burden was determined by plating of serial dilutions on $7 \mathrm{H} 11$ plates.

Detection of nitrites by the Griess reaction. Culture supernatants were assessed for nitrite production by using the Griess Reagent System Kit (Promega), according to the manufacturer's instructions.

Statistics. Differences between the means of multiple experimental groups were analyzed using one-way ANOVA with Tukey's posthoc test. For all other analyses, we used the 2-tailed Student's $t$ test. Correlation between variables was determined by calculating Pearson's coefficient using a 2-tailed analysis. Differences were considered significant when $P \leq 0.05$. For all figures, data represent mean $\pm S D$. All analyses were performed using GraphPad Prism Software.

Study approval. All individuals were examined as part of a clinical research protocol approved by the Institutional Review Board of the Tuberculosis Outpatient Clinic, INER, or NIH-NIRT-ICERT, and informed written consent was obtained from all participants. All mice were used following the NIH guidelines for housing and care of laboratory animals and in accordance with University of Pittsburgh and Washington University in St. Louis Institutional Animal Care and Use Committee guidelines. All efforts were made to minimize suffering and pain, as described in these approved protocols.

\section{Author contributions}

LM, KLG, WYL, RG, MA, AR, ACL, BAR, RRP, and JRM performed the experiments; LM, KLG, PJM, JKK, EJP, and SAK designed the experiments; LM, KLG, RG, DDK, JRM, JZ, SB, MM, TEM, PJM, JKK, and SAK carried out analyses; LM and SAK wrote the paper; and LM, KLG, JKK, PJM, EJP, and SAK edited the paper. SAK, EJP, $\mathrm{TEM}$, and JRM provided funding.

\section{Acknowledgments}

This work was supported by Washington University in St. Louis, Children's Hospital of Pittsburgh, NIH grants HL105427 to S.A. Khader, AI032573 to E.J. Pearce, and AI108725 to T.E. Morrison. L. Monin was supported by Children's Hospital of Pittsburgh Research Advisory Committee Grant from Children's Hospital of Pittsburgh of the UPMC Health System. K.L. Griffiths was supported by American Lung Association Senior Research Training Fellowship RT-30592. J. Rangel-Moreno was supported by funds of the Department of Medicine, University of Rochester, and U19 AI91036. The authors thank Keke Fairfax and Amber Smith for SEA preparation and Markus Mohrs for providing IFN- $\gamma$-YFP reporter mice. We thank Nathella Pavan Kumar, R. Sridhar, and V.V. Banurekha for assistance with recruitment of patients.

Address correspondence to: Shabaana A. Khader, Department of Molecular Microbiology, Campus Box 8230, 660 South Euclid Avenue, St. Louis, Missouri 63110-1093, USA. Phone: 314.286.1590; E-mail: khader@wustl.edu.
1. Dye C, Glaziou P, Floyd K, Raviglione M. Prospects for tuberculosis elimination. Anпи Rev Public Health. 2013;34:271-286.

2. Cooper AM, Khader SA. The role of cytokines in the initiation, expansion, and control of cel- lular immunity to tuberculosis. Immunol Rev. 2008;226:191-204.

3. Maizels RM, Balic A, Gomez-Escobar N, Nair M, Taylor MD, Allen JE. Helminth parasites - masters of regulation. Immunol Rev. 2004;201:89-116.
4. Diaz A, Allen JE. Mapping immune response profiles: the emerging scenario from helminth immunology. Eur J Immunol. 2007;37(12):3319-3326.

5. Rafi W, Ribeiro-Rodrigues R, Ellner JJ, Salgame P. 'Coinfection-helminthes and tuberculosis'. 
Curr Opin HIV AIDS. 2012;7(3):239-244.

6. Hotez PJ, Brindley PJ, Bethony JM, King CH, Pearce EJ, Jacobson J. Helminth infections: the great neglected tropical diseases. J Clin Invest. 2008;118(4):1311-1321.

7. Elias D, Wolday D, Akuffo H, Petros B, Bronner U, Britton S. Effect of deworming on human $\mathrm{T}$ cell responses to mycobacterial antigens in helminthexposed individuals before and after bacille Calmette-Guerin (BCG) vaccination. Clin Exp Immunol. 2001;123(2):219-225.

8. Elias D, Akuffo H, Britton S. Helminthes could influence the outcome of vaccines against TB in the tropics. Parasite Immunol. 2006;28(10):507-513.

9. Elias D, Britton S, Aseffa A, Engers H, Akuffo H. Poor immunogenicity of BCG in helminth infected population is associated with increased in vitro TGF- $\beta$ production. Vaccine. 2008;26(31):3897-3902.

10. Brown M, et al. Schistosoma mansoni, nematode infections, and progression to active tuberculosis among HIV-1-infected Ugandans. Am J Trop Med Hyg. 2006;74(5):819-825.

11. Elias D, Akuffo H, Thors C, Pawlowski A, Britton S. Low dose chronic Schistosoma mansoni infection increases susceptibility to Mycobacterium bovis BCG infection in mice. Clin Exp Immunol. 2005;139(3):398-404.

12. Elias D, Akuffo H, Pawlowski A, Haile M, Schon $\mathrm{T}$, Britton S. Schistosoma mansoni infection reduces the protective efficacy of BCG vaccination against virulent Mycobacterium tuberculosis. Vaccine. 2005;23(11):1326-1334.

13. Slight SR, et al. CXCR5 ${ }^{+} \mathrm{T}$ helper cells mediate protective immunity against tuberculosis. J Clin Invest. 2013;123(2):712-726.

14. Gopal R, et al. S100A8/A9 proteins mediate neutrophilic inflammation and lung pathology during tuberculosis. Am J Respir Crit Care Med. 2013;188(9):1137-1146.

15. Pearce EJ, MacDonald AS. The immunobiology of schistosomiasis. Nat Rev Immunol. 2002;2(7):499-511.

16. Cooper AM, Dalton DK, Stewart TA, Griffin JP, Russell DG, Orme IM. Disseminated tuberculosis in interferon gamma gene-disrupted mice. JExp Med.1993;178(6):2243-2247.
17. Liu R, Dong HF, Guo Y, Zhao QP, Jiang MS. Efficacy of praziquantel and artemisinin derivatives for the treatment and prevention of human schistosomiasis: a systematic review and meta-analysis. Parasit Vectors. 2011;4:201.

18. El Kasmi KC, et al. Toll-like receptor-induced arginase 1 in macrophages thwarts effective immunity against intracellular pathogens. Nat Immunol. 2008;9(12):1399-1406.

19. Pesce JT, et al. Arginase-1-expressing macrophages suppress Th2 cytokine-driven inflammation and fibrosis. PLoS Pathog. 2009;5(4):e1000371.

20. Barron L, et al. Role of arginase 1 from myeloid cells in th2-dominated lung inflammation. PLoS One. 2013;8(4):e61961.

21. Duque-Correa MA, et al. Macrophage arginase-1 controls bacterial growth and pathology in hypoxic tuberculosis granulomas. Proc Natl Acad Sci U S A. 2014;111(38):E4024-E4032.

22. Bowcutt R, et al. Arginase-1-expressing macrophages are dispensable for resistance to infection with the gastrointestinal helminth Trichuris muris. Parasite Immunol. 2011;33(7):411-420.

23. Kang DD, Lin Y, Moreno JR, Randall TD, Khader SA. Profiling early lung immune responses in the mouse model of tuberculosis. PLoS One. 2011;6(1):e16161.

24. Scanga C, Mohan V, Joseph H, Yu K, Chan J, Flynn J. Reactivation of latent tuberculosis: variations on the Cornell murine model. Infect Immun. 1999;67(9):4531-4538.

25. Nouailles G, et al. CXCL5-secreting pulmonary epithelial cells drive destructive neutrophilic inflammation in tuberculosis. JClin Invest. 2014;124(3):1268-1282.

26. Murray PJ, Wynn TA. Protective and pathogenic functions of macrophage subsets. Nat Rev Immunol. 2011;11(11):723-737.

27. Potian JA, Rafi W, Bhatt K, McBride A, Gause WC, Salgame P. Preexisting helminth infection induces inhibition of innate pulmonary antituberculosis defense by engaging the IL-4 receptor pathway. J Exp Med. 2011;208(9):1863-1874.

28. Erb KJ, Trujillo C, Fugate M, Moll H. Infection with the helminth Nippostrongylus brasiliensis does not interfere with efficient elimination of Mycobacterium bovis BCG from the lungs of mice. Clin
Diagn Lab Immunol. 2002;9(3):727-730.

29. Rafi W, Bhatt K, Gause WC, Salgame P. "Primary memory immunity to Mycobacterium tuberculosis infection is not compromised in mice with chronic enteric helminth infection". Infect Immun. 2015;83(3):1217-1223.

30. Babu S, et al. Human type 1 and 17 responses in latent tuberculosis are modulated by coincident filarial infection through cytotoxic T lymphocyte antigen-4 and programmed death-1.J Infect Dis. 2009;200(2):288-298.

31. Mattila JT, et al. Microenvironments in tuberculous granulomas are delineated by distinct populations of macrophage subsets and expression of nitric oxide synthase and arginase isoforms. J Immunol. 2013;191(2):773-784.

32. Pessanha AP, Martins RA, Mattos-Guaraldi AL, Vianna A, Moreira LO. Arginase-1 expression in granulomas of tuberculosis patients. FEMS Immunol Med Microbiol. 2012;66(2):265-268.

33. Maarsingh H, Pera T, Meurs H. Arginase and pulmonary diseases. Naunyn Schmiedebergs Arch Pharmacol. 2008;378(2):171-184.

34. Bisoffi $Z$, et al. Diagnostic accuracy of five serologic tests for Strongyloides stercoralis infection. PLoS Negl Trop Dis. 2014;8(1):e2640.

35. Mayer K, et al. The functional heterogeneity of type I effector $\mathrm{T}$ cells in response to infection is related to the potential for IFN- $\gamma$ production. JImmunol. 2005;174(12):7732-7739.

36. Reiley WW, et al. ESAT-6-specific CD 4 T cell responses to aerosol Mycobacterium tuberculosis infection are initiated in the mediastinal lymph nodes. Proc Natl Acad Sci U S A. 2008;105(31):10961-10966.

37. Zaretsky AG, Taylor JJ, King IL, Marshall FA, Mohrs M, Pearce EJ. T follicular helper cells differentiate from Th2 cells in response to helminth antigens. JExp Med. 2009;206(5):991-999.

38. Crosby A, et al. Praziquantel reverses pulmonary hypertension and vascular remodeling in murine schistosomiasis. Am J Respir Crit Care Med. 2011;184(4):467-473.

39. Khader SA, et al. IL-23 and IL-17 in the establishment of protective pulmonary $\mathrm{CD} 4^{+} \mathrm{T}$ cell responses after vaccination and during Mycobacterium tuberculosis challenge. Nat Immunol. 2007;8(4):369-377. 